\title{
Novel Moraxella catarrhalis prophages display hyperconserved non-structural genes despite their genomic diversity
}

\author{
Amir Ariff' ${ }^{1}$, Michael J. Wise ${ }^{2}$, Charlene M. Kahler ${ }^{1}$, Chin Yen Tay ${ }^{1}$, Fanny Peters ${ }^{1}$, Timothy T. Perkins ${ }^{1+}$
} and Barbara J. Chang ${ }^{1 *+}$

\begin{abstract}
Background: Moraxella catarrhalis is an important pathogen that often causes otitis media in children, a disease that is not currently vaccine preventable. Asymptomatic colonisation of the human upper respiratory tract is common and lack of clearance by the immune system is likely due to the emergence of seroresistant genetic lineages. No active bacteriophages or prophages have been described in this species. This study was undertaken to identify and categorise prophages in $M$. catarrhalis, their genetic diversity and the relationship of such diversity with the host-species phylogeny.
\end{abstract}

Results: This study presents a comparative analysis of 32 putative prophages identified in 95 phylogenetically variable, newly sequenced $M$. catarrhalis genomes. The prophages were genotypically classified into four diverse clades. The genetic synteny of each clade is similar to the group 1 phage family Siphoviridae, however, they form genotypic clusters that are distinct from other members of this family. No core genetic sequences exist across the 32 prophages despite clades 2, 3, and 4 sharing the most sequence identity. The analysis of non-structural prophage genes (coding the integrase, and terminase), and portal gene showed that the respective genes were identical for clades 2, 3, and 4, but unique for clade 1. Empirical analysis calculated that these genes are unexpectedly hyperconserved, under purifying selection, suggesting a tightly regulated functional role. As such, it is improbable that the prophages are decaying remnants but stable components of a fluctuating, flexible and unpredictable system ultimately maintained by functional constraints on non-structural and packaging genes. Additionally, the plate encoding genes were well conserved across all four prophage clades, and the tail fibre genes, commonly responsible for receptor recognition, were clustered into three major groups distributed across the prophage clades. A pan-genome of 283,622 bp was identified, and the prophages were mapped onto the diverse M. catarrhalis multi-locus sequence type (MLST) backbone.

Conclusion: This study has provided the first evidence of putatively mobile prophages in $M$. catarrhalis, identifying a diverse and fluctuating system dependent on the hyperconservation of a few key, non-structural genes. Some prophages harbour virulence-related genes, and potentially influence the physiology and virulence of $M$. catarrhalis. Importantly our data will provide supporting information on the identification of novel prophages in other species by adding greater weight to the identification of non-structural genes.

Keywords: Moraxella catarrhalis, Prophages, Bacteriophages, Siphoviridae, Pan-genome, Multi-locus sequence typing, Phage-related genes, Hyperconservation, Gram-negative diplococcus, Non-structural genes

\footnotetext{
* Correspondence: barbara.chang@uwa.edu.au

†Equal contributors

${ }^{1}$ School of Pathology and Laboratory Medicine, The University of Western

Australia, Perth, WA, Australia

Full list of author information is available at the end of the article
}

\section{Biomed Central}

(c) 2015 Ariff et al. Open Access This article is distributed under the terms of the Creative Commons Attribution 4.0 International License (http://creativecommons.org/licenses/by/4.0/), which permits unrestricted use, distribution, and reproduction in any medium, provided you give appropriate credit to the original author(s) and the source, provide a link to the Creative Commons license, and indicate if changes were made. The Creative Commons Public Domain Dedication waiver (http://creativecommons.org/publicdomain/zero/1.0/) applies to the data made available in this article, unless otherwise stated. 


\section{Background}

M. catarhalis is a commensal and important pathogen of the human upper respiratory tract and middle ear. After Streptococcus pyogenes and Haemophilus influenzae, $M$. catarrhalis is the leading cause of upper respiratory tract and middle ear infections in humans, commonly presenting as otitis media in children below 2 years of age [1], and as a variety of diseases in adults and the elderly, ranging from chronic obstructive pulmonary disease (COPD), to pneumonia, bronchitis, laryngitis, and sinusitis [2]. Infrequently, $M$. catarrhalis can cause septic arthritis, bacteraemia [3], endocarditis [4], meningitis [5], and other invasive infections [2]. M. catarrhalis colonises the upper respiratory tracts of up to $75 \%$ of children, though the colonisation rate reduces to approximately $1 \%$ in adults [2]. $M$. catarrhalis has a range of virulence factors, which allow the bacterium to adhere to host epithelial cells, enter host tissues, successfully multiply, interfere with and avoid host defence mechanisms, and cause disease [2]. The virulence of $M$. catarrhalis is well described, particularly regarding the mechanisms of serum-resistance [6-8]. The characterisation of virulence factors has been associated with two major clades of the bacterium: 1) a serum-resistant clade that has a higher representation of virulence factors, is complement-resistant, has increased adhesion to epithelial cells, displays higher genomic diversity, and has a more recent evolution of about $5 \mathrm{Ma}$ ago; and 2) a serum-sensitive clade, which has the converse traits [9].

Bacteriophages are viruses that infect bacteria and utilise the host's cell machinery to replicate and propagate. Two lifecycles of phages are defined: lytic phages, which contain a copy of the phage genome packaged in its capsid that is built into its quaternary structure prior to lysing the host cell and subsequent release; and lysogenic or temperate phages, which may opt instead to integrate into the host cell's genome, lying dormant until conditions are suitable to re-enter the lytic pathway [10]. Temperate phages that are integrated into the host genome are known as prophages. Prophages may harbour cargo genes, which are non-essential for phage function, but may confer virulence and other traits to the host cell. This may benefit either or both the phage and host bacterium, and is well categorised in the case of toxins, cell adhesion molecules, nutrient uptake, immune response evasion, fimbriae and others [11, 12]. While integrated into the host genome, prophages undergo evolutionary pressures different from those undergone by phage particles, leading to host-prophage driven selection and genetic flux, even within prophage genes that do not affect host physiology [13, 14]. The rapid increase in bacterial genome sequencing has led to the identification of numerous prophages [15] and has furthered our understanding of phage roles in host physiology and pathology of disease [16].
Active phages are often difficult to identify due to their size and sporadic signals that lead to lytic activation. Scant phage-associated open reading frames (ORFs) have been described in $M$. catarrhalis genomes [15], however, no active phages or complete prophages have yet been identified in the species. As phages may contribute in important ways to the survival and pathogenesis of $M$. catarrhalis, we aimed to screen a diverse collection of M. catarhalis isolates for the presence of prophages and to characterise these genetic elements. This study utilised a bioinformatics approach, resulting in the identification of 32 novel $M$. catarrhalis prophages. Analysis of the prophage pan-genome and phage-related genes supported the categorisation of prophages into four clades, where clades 2,3 , and 4 share regions of homogeneity and clade 1 was unique. Further analysis of the phage-related genes showed a relationship with known double-stranded DNA, tailed phages, the Siphoviridae. The non-structural genes encoding the integrase and terminase, as well as a structural gene encoding the portal protein were hyperconserved despite the surrounding diversity of the identified prophages. Additionally, several virulence-related factors were identified, which may suggest a role of these prophages in virulence of $M$. catarrhalis.

\section{Results}

\section{Identification of putative $M$. catarrhalis prophages}

PHAST identified 32 putative complete prophages, 54 questionable prophages, and 131 incomplete prophages in $95 \mathrm{M}$. catarrhalis genomes (Table 1 and Additional file 1: Figure S1.; see methods for classification criteria). Each of the 32 putative complete prophages was found in a different strain of $M$. catarrhalis, and the strains O35E and 2041717D harboured no prophage of any category.

The putative complete prophages ranged between $25 \mathrm{~kb}$ to $55 \mathrm{~kb}$ in length, with a median length of approximately $40 \mathrm{~kb}$. The GC content of the prophages ranged between 41.40 and $44.14 \%$, with an average GC content of $42.90 \%$ - higher than the average GC content for the M. catarrhalis host of $41 \%$ [17]. Twenty-three putative attachment sites were identified amongst 20 prophages (Table 1).

\section{Classification and similarity of $M$. catarrhalis prophages}

The progressiveMauve blocks alignment categorised the complete putative prophages into 4 distinct clades (Fig. 1). The addition of archetypes from all genera of Caudovirales bacteriophages to the distance tree revealed that $M$. catarrhalis prophages are related to, but distinct from the following genera of Siphoviridae: $\lambda$-like viruses, L5-like viruses, N15-like viruses, $\phi$ C31-like viruses, and Tuna-like viruses. The archetypes from each 
Table 1 Strains analysed in this study

\begin{tabular}{|c|c|c|c|c|c|c|c|c|c|}
\hline \multirow[t]{2}{*}{ Strain } & \multirow[t]{2}{*}{ Source ${ }^{a}$} & \multirow[t]{2}{*}{$\begin{array}{l}\text { Clinical/ } \\
\text { Commensal }\end{array}$} & \multirow[t]{2}{*}{$\mathrm{MLST}^{\mathrm{b}}$} & \multicolumn{3}{|c|}{$\begin{array}{l}\text { Phage } \\
\text { presence }^{c}\end{array}$} & \multirow{2}{*}{$\begin{array}{l}\text { Phage } \\
\text { name } \\
(\% G C)\end{array}$} & \multirow[t]{2}{*}{ att site ${ }^{e}$} & \multirow{2}{*}{$\begin{array}{l}\text { NCBI } \\
\text { prophage } \\
\text { accession } \\
\text { number }\end{array}$} \\
\hline & & & & I & Q & $C$ & & & \\
\hline $2019228 G$ & PathWest & Clinical & $\mathrm{h}$ & 1 & 0 & 0 & & & \\
\hline $1020848 \mathrm{M}$ & PathWest & Clinical & 11 & 2 & 0 & 1 & $\begin{array}{l}\text { Mcat7 } \\
\text { (43.62 \%) }\end{array}$ & $\begin{array}{l}\text { 5'-ATCAAAAAATGG-3' } \\
\text { 5'-AATCAAAAATCT-3' } \\
\text { 5'-TTITTATTGGG-3' }\end{array}$ & KR093631 \\
\hline $1028680 \mathrm{~K}$ & PathWest & Clinical & 41 & 1 & 0 & 0 & & & \\
\hline 1034084Q & PathWest & Clinical & 224 & 1 & 0 & 0 & & & \\
\hline 2023641 W & PathWest & Clinical & 4 & 2 & 1 & 0 & & & \\
\hline $3331584 D$ & PathWest & Clinical & h & 0 & 1 & 0 & & & \\
\hline $3476642 E$ & PathWest & Clinical & 46 & 1 & 1 & 0 & & & \\
\hline $3481088 Y$ & PathWest & Clinical & h & 2 & 3 & 0 & & & \\
\hline $3753746 B$ & PathWest & Clinical & h & 0 & 0 & 1 & $\begin{array}{l}\text { Mcat9 } \\
(43.94 \%)\end{array}$ & 5'-TGTGTACATAATTGTGTACATA-3' & KR093633 \\
\hline $4789849 \mathrm{~F}$ & PathWest & Clinical & 67 & 1 & 1 & 0 & & & \\
\hline $5550565 E$ & PathWest & Clinical & h & 3 & 1 & 1 & $\begin{array}{l}\text { Mcat32 } \\
\text { (42.68 \%) }\end{array}$ & N/A & KR093656 \\
\hline 20236154 & PathWest & Clinical & 236 & 1 & 0 & 0 & & & \\
\hline 20370737 & PathWest & Clinical & 105 & 1 & 0 & 1 & $\begin{array}{l}\text { Mcat4 } \\
\text { (43.21\%) }\end{array}$ & 5'-TTATCAATCAGT-3' & KR093628 \\
\hline 2040048B & PathWest & Clinical & 230 & 0 & 1 & 0 & & & \\
\hline $3503282 R$ & PathWest & Clinical & 184 & 2 & 0 & 1 & $\begin{array}{l}\text { Mcat16 } \\
\text { (42.96\%) }\end{array}$ & $5^{\prime}-T \pi T T T A G G G G-3^{\prime}$ & KR093640 \\
\hline $5004663 G$ & PathWest & Clinical & 224 & 1 & 0 & 0 & & & \\
\hline $1093063 Y$ & PathWest & Clinical & 230 & 0 & 1 & 0 & & & \\
\hline $4640032 P$ & PathWest & Clinical & 246 & 1 & 0 & 1 & $\begin{array}{l}\text { Mcat15 } \\
\text { (42.71\%) }\end{array}$ & 5'-ATACAAAAAATC-3' & KR093639 \\
\hline $2046210 Y$ & PathWest & Clinical & h & 2 & 0 & 0 & & & \\
\hline 4737718Q & PathWest & Clinical & h & 2 & 0 & 0 & & & \\
\hline $5012204 D$ & PathWest & Clinical & 250 & 4 & 2 & 1 & $\begin{array}{l}\text { Mcat24 } \\
(42.74 \%)\end{array}$ & N/A & KR093648 \\
\hline $4840991 \mathrm{~N}$ & PathWest & Clinical & 73 & 1 & 0 & 0 & & & \\
\hline $2042044 P$ & PathWest & Clinical & h & 0 & 0 & 0 & & & \\
\hline 2041717D & PathWest & Clinical & h & 0 & 0 & 0 & & & \\
\hline $5008863 \mathrm{~L}$ & PathWest & Clinical & h & 1 & 1 & 1 & $\begin{array}{l}\text { Mcat19 } \\
\text { (42.39\%) }\end{array}$ & 5'-ATTाTाTATATT-3' & KR093643 \\
\hline $1583718 S$ & PathWest & Clinical & 234 & 2 & 1 & 0 & & & \\
\hline $5267783 B$ & PathWest & Clinical & 234 & 3 & 1 & 0 & & & \\
\hline 4431503 J & PathWest & Clinical & 118 & 3 & 2 & 0 & & & \\
\hline $1111988 \mathrm{H}$ & PathWest & Clinical & h & 1 & 2 & 0 & & & \\
\hline $5021467 Y$ & PathWest & Clinical & 224 & 1 & 0 & 0 & & & \\
\hline $5560626 \mathrm{Q}$ & PathWest & Clinical & h & 1 & 0 & 0 & & & \\
\hline $2047127 \mathrm{~K}$ & PathWest & Clinical & 240 & 1 & 0 & 0 & & & \\
\hline $5021466 \mathrm{~N}$ & PathWest & Clinical & 224 & 1 & 0 & 0 & & & \\
\hline $2041417 P$ & PathWest & Clinical & h & 3 & 1 & 1 & $\begin{array}{l}\text { Mcat3 } \\
\text { (43.79 \%) }\end{array}$ & 5'-AAAAAAATCAAAG-3' & KR093627 \\
\hline 1098655R & PathWest & Clinical & 230 & 1 & 1 & 0 & & & \\
\hline
\end{tabular}


Table 1 Strains analysed in this study (Continued)

\begin{tabular}{|c|c|c|c|c|c|c|c|c|c|}
\hline $5157102 Y$ & PathWest & Clinical & 251 & 1 & 0 & 0 & & & \\
\hline 4849094R & PathWest & Clinical & 248 & 1 & 0 & 0 & & & \\
\hline $2050675 Y$ & PathWest & Clinical & h & 1 & 0 & 0 & & & \\
\hline $5553245 S$ & PathWest & Clinical & h & 1 & 0 & 0 & $\begin{array}{l}\text { Mcat13 } \\
(42.77 \%)\end{array}$ & $5^{\prime}-T T T T C A G C T T C-3^{\prime}$ & KR093637 \\
\hline $5 / 131 / 1$ & KOMRP & Commensal & 217 & 1 & 0 & 0 & & & \\
\hline 23/41/1 & KOMRP & Commensal & 235 & 2 & 0 & 0 & & & \\
\hline $39 / 355 / 1$ & KOMRP & Commensal & 62 & 1 & 1 & 1 & $\begin{array}{l}\text { Mcat2 } \\
(43.64 \%)\end{array}$ & $5^{\prime}$-TTTCAAATTTTA-3' & KR093626 \\
\hline $41 / 539 / 1$ & KOMRP & Commensal & h & 1 & 0 & 1 & $\begin{array}{l}\text { Mcat22 } \\
(42.48 \%)\end{array}$ & 5'-AAAAATTTGGTT-3' & KR093646 \\
\hline $3 / 7 / 1$ & KOMRP & Commensal & 242 & 1 & 1 & 0 & & & \\
\hline $102 / 402 / 3$ & KOMRP & Commensal & h & 1 & 3 & 0 & & & \\
\hline $91 / 291 / 2$ & KOMRP & Commensal & h & 2 & 0 & 1 & $\begin{array}{l}\text { Mcat20 } \\
(42.71 \%)\end{array}$ & N/A & KR093644 \\
\hline 78/191/1 & KOMRP & Commensal & h & 4 & 0 & 1 & $\begin{array}{l}\text { Mcat31 } \\
(42.68 \%)\end{array}$ & N/A & KR093655 \\
\hline $96 / 281 / 2$ & KOMRP & Commensal & 259 & 1 & 0 & 0 & & & \\
\hline 26/133/1 & KOMRP & Commensal & 70 & 2 & 0 & 1 & $\begin{array}{l}\text { Mcat23 } \\
(42.65 \%)\end{array}$ & N/A & KR093647 \\
\hline $129 / 414 / 4$ & KOMRP & Commensal & 209 & 1 & 0 & 0 & & & \\
\hline $130 / 563 / 4$ & KOMRP & Commensal & 74 & 2 & 0 & 0 & & & \\
\hline $60 / 120 / 1$ & KOMRP & Commensal & 64 & 1 & 0 & 1 & $\begin{array}{l}\text { Mcat11 } \\
(43.92 \%)\end{array}$ & 5'-TAAAAAAAATAAA-3' & KR093635 \\
\hline $105 / 305 / 2$ & KOMRP & Commensal & h & 0 & 1 & 0 & & & \\
\hline $113 / 391 / 3$ & KOMRP & Commensal & 232 & 1 & 0 & 0 & & & \\
\hline 20/122/1 & KOMRP & Commensal & 235 & 2 & 0 & 0 & & & \\
\hline $79 / 220 / 2$ & KOMRP & Commensal & h & 1 & 2 & 0 & & & \\
\hline 29/50/1 & KOMRP & Commensal & 241 & 0 & 1 & 0 & & & \\
\hline 23/95/1 & KOMRP & Commensal & 233 & 1 & 0 & 0 & & & \\
\hline $107 / 374 / 3$ & KOMRP & Commensal & 229 & 2 & 0 & 0 & & & \\
\hline $76 / 204 / 3$ & KOMRP & Commensal & h & 1 & 0 & 0 & & & \\
\hline 73/187/1 & KOMRP & Commensal & 254 & 0 & 1 & 1 & $\begin{array}{l}\text { Mcat10 } \\
(44.14 \%)\end{array}$ & $5^{\prime}$-TAAAAAAAATAAA-3' & KR093634 \\
\hline 24/92/1 & KOMRP & Commensal & 64 & 0 & 0 & 1 & $\begin{array}{l}\text { Mcat12 } \\
(43.56 \%)\end{array}$ & 5'-TTATTाTAAAAA-3' & KR093636 \\
\hline $78 / 325 / 3$ & KOMRP & Commensal & 256 & 2 & 0 & 0 & & & \\
\hline $78 / 205 / 2$ & KOMRP & Commensal & h & 5 & 0 & 1 & $\begin{array}{l}\text { Mcat30 } \\
(42.68 \%)\end{array}$ & N/A & KR093654 \\
\hline $105 / 417 / 3$ & KOMRP & Commensal & h & 1 & 1 & 0 & & & \\
\hline $25 / 44 / 1$ & KOMRP & Commensal & h & 1 & 1 & 0 & & & \\
\hline $77 / 338 / 2$ & KOMRP & Commensal & 50 & 0 & 2 & 0 & & & \\
\hline $16 / 38 / 1$ & KOMRP & Commensal & 157 & 1 & 0 & 1 & $\begin{array}{l}\text { Mcat26 } \\
(42.22 \%)\end{array}$ & N/A & KR093650 \\
\hline $64 / 108 / 1$ & KOMRP & Commensal & 140 & 0 & 2 & 1 & $\begin{array}{l}\text { Mcat17 } \\
(42.46 \%)\end{array}$ & $\begin{array}{l}\text { 5'-ACCATTTITAA-3' } \\
5^{\prime} \text {-TIIIITCATIT-3' }\end{array}$ & KR093641 \\
\hline $3 / 22 / 1$ & KOMRP & Commensal & 242 & 1 & 0 & 0 & & & \\
\hline $80 / 196 / 2$ & KOMRP & Commensal & h & 2 & 1 & 0 & & & \\
\hline
\end{tabular}


Table 1 Strains analysed in this study (Continued)

\begin{tabular}{|c|c|c|c|c|c|c|c|c|c|}
\hline $15 / 36 / 1$ & KOMRP & Commensal & 233 & 1 & 0 & 0 & & & \\
\hline $\mathrm{BBH} 18$ & $\begin{array}{l}\text { Sputum sample, patient with COPD, } \\
\text { Holland GCA_000092265.1 }\end{array}$ & Clinical & 128 & 1 & 0 & 0 & & & \\
\hline \multirow[t]{2}{*}{ A16 } & \multirow[t]{2}{*}{ Frozen stocks, saliva, Australia } & \multirow[t]{2}{*}{ Commensal } & \multirow[t]{2}{*}{ h } & \multirow[t]{2}{*}{0} & \multirow[t]{2}{*}{0} & \multirow[t]{2}{*}{0} & \multirow{2}{*}{$\begin{array}{l}\text { Mcat28 } \\
(42.24 \%)\end{array}$} & 5'-TGTGTACATAATTGTGTACATA-3' & \multirow[t]{2}{*}{ KR093652 } \\
\hline & & & & & & & & 5'-AAAAAACTTAAC-3' & \\
\hline ATCC43617 & $\begin{array}{l}\text { Trans-tracheal aspirate from coal miner } \\
\text { with bronchitis }\end{array}$ & Clinical & 25 & 1 & 1 & 1 & $\begin{array}{l}\text { Mcat5 } \\
(43.30 \%)\end{array}$ & N/A & KR093629 \\
\hline ATCC43617a ${ }^{f}$ & $\begin{array}{l}\text { Trans-tracheal aspirate from coal miner } \\
\text { with bronchitis }\end{array}$ & Clinical & 25 & 1 & 1 & 1 & $\begin{array}{l}\text { Mcat6 } \\
(43.08 \%)\end{array}$ & N/A & KR093630 \\
\hline BE4L & PathWest & Clinical & h & 2 & 0 & 1 & $\begin{array}{l}\text { Mcat25 } \\
(42.61 \%)\end{array}$ & & \\
\hline MC4S & Alexander project & Clinical & 188 & 1 & 2 & 1 & $\begin{array}{l}\text { Mcat8 } \\
(41.40 \%)\end{array}$ & 5'-CTTAAAAAAATA-3' & KR093632 \\
\hline MC24 & Alexander project & Clinical & h & 1 & 0 & 0 & & & \\
\hline MC1 & Alexander project & Clinical & 131 & 1 & 0 & 0 & & & \\
\hline BE5 & PathWest & Clinical & $\mathrm{h}$ & 1 & 1 & 1 & $\begin{array}{l}\text { Mcat18 } \\
(42.26 \%)\end{array}$ & 5'-CATTAATCAAAT-3' & KR093642 \\
\hline A6 & Frozen stocks, saliva, Australia & Commensal & 3 & 6 & 0 & 1 & $\begin{array}{l}\text { Mcat29 } \\
(42.23 \%)\end{array}$ & N/A & KR093653 \\
\hline T6 & Sputum, children $3-6$ years, Taiwan & Commensal & h & 1 & 1 & 0 & & & \\
\hline $\mathrm{T} 12$ & Sputum, children $3-6$ years, Taiwan & Commensal & h & 2 & 1 & 0 & & & \\
\hline T4 & Sputum, children 3 - 6 years, Taiwan & Commensal & 191 & 1 & 0 & 0 & & & \\
\hline K117 & Hospital isolate, Perth, Australia & Clinical & 40 & 1 & 0 & 0 & & & \\
\hline $12 \mathrm{P} 80 \mathrm{~B} 1^{\mathrm{g}}$ & GCA_000192965.2 & Clinical & 185 & 1 & 0 & 0 & & & \\
\hline $\mathrm{O} 35 \mathrm{E}^{\mathrm{g}}$ & GCA_000193085.2 & Clinical & 146 & 0 & 0 & 0 & & & \\
\hline $46 \mathrm{P} 47 \mathrm{~B} 1^{9}$ & GCA_000192945.2 & Clinical & h & 1 & 0 & 1 & $\begin{array}{l}\text { Mcat21 } \\
(42.26 \%)\end{array}$ & N/A & KR093645 \\
\hline $101 \mathrm{P} 10 \mathrm{~B} 1^{\mathrm{g}}$ & GCA_000193065.2 & Clinical & 218 & 1 & 1 & 0 & & & \\
\hline $103 \mathrm{P} 14 \mathrm{~B} 1^{9}$ & GCA_000192925.2 & Clinical & 187 & 1 & 1 & 1 & $\begin{array}{l}\text { Mcat14 } \\
(43.39 \%)\end{array}$ & N/A & KR093638 \\
\hline $7169^{9}$ & GCA_000192905.2 & Clinical & 82 & 3 & 2 & 0 & & & \\
\hline $\mathrm{BC} 1^{\mathrm{g}}$ & GCA_0000192985.2 & Clinical & 216 & 2 & 1 & 0 & & & \\
\hline$B C 7^{9}$ & GCA_000193005.2 & Clinical & 217 & 1 & 0 & 0 & & & \\
\hline $\mathrm{BC} 8^{\mathrm{g}}$ & GCA_0000193025.2 & Clinical & 162 & 1 & 1 & 1 & $\begin{array}{l}\text { Mcat1 } \\
(43.62 \%)\end{array}$ & 5'-TTTCAAATTTTA-3' & KR093625 \\
\hline $\mathrm{CO}^{\mathrm{g}}$ & GCA_000193045.2 & Clinical & 199 & 2 & 0 & 1 & $\begin{array}{l}\text { Mcat27 } \\
(42.57 \%)\end{array}$ & 5'-TGTGTACATAATTGTGTACATA-3' & KR093651 \\
\hline $\mathrm{RH} 4^{\mathrm{g}}$ & GCA_000302495.1 & Clinical & h & 0 & 0 & 1 & & & \\
\hline
\end{tabular}

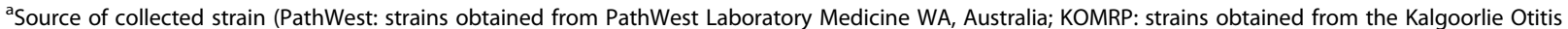
Media Research Project [49])

${ }^{b}$ Multi locus sequence typing strain types

'Phage presence as annotated by PHAST, where I = incomplete prophage, $\mathrm{Q}=$ questionable prophage, and $\mathrm{C}=$ complete prophage

${ }^{\mathrm{d}}$ Complete prophages were named Mcat1 to Mcat32

${ }^{e}$ att sites are putative flanking attachment sites identified in $M$. catarrhalis genomes by PHAST. Prophages without identified att sites are labelled N/A

${ }^{\mathrm{f}}$ The strain ATCC43617a is a passaged derivative of ATCC43617 that was obtained from The University of Queensland and sequenced in this study. Additionally, the sequenced genome of ATCC43617 available on the NCBI database was also analysed [15]

${ }^{9}$ Strains not sequenced in this study, but are available on the NCBI database; GenBank accession numbers current at time of manuscript submission [15]

${ }^{h}$ denotes novel strain types

of these five genera formed a clade separate from the $M$. catarrhalis prophages. There was no significant identity between the $M$. catarrhalis prophages and the phages of the families Podoviridae or Myoviridae (data not shown).
Additionally, the regions with greatest sequence identity to the listed five genera of Siphoviridae, comprised the tail fibre genes. This result was further confirmed by the Virfam analysis, which categorised all prophages as 


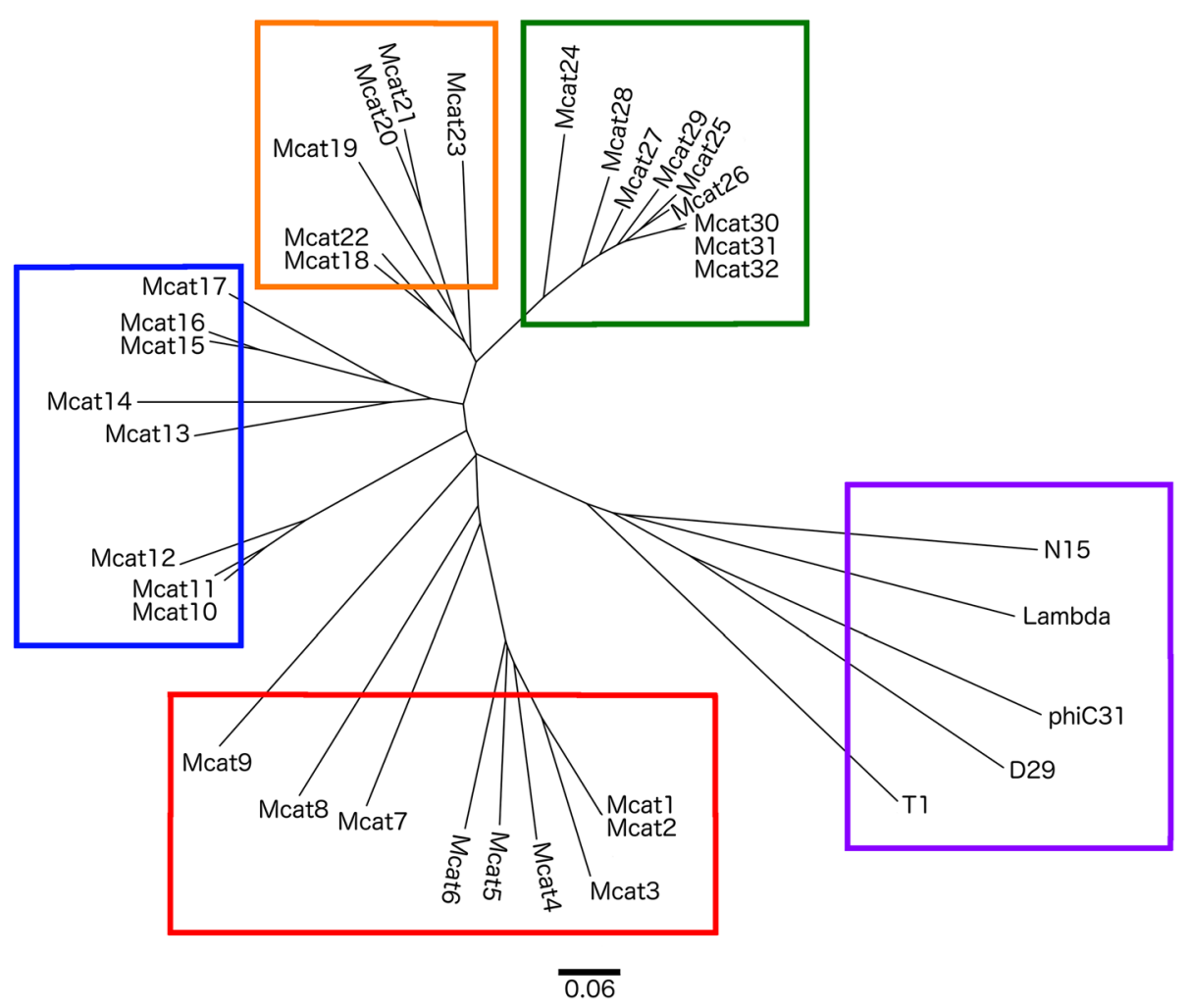

Fig. 1 Prophages of Moraxella catarrhalis are divided into four clades, grouped by nucleotide similarity. The 32 prophages identified in 95 sequences of $M$. catarrhalis were aligned using progressiveMauve and compared to five reference Siphoviridae genera: $\lambda$-like, L5-like, N15-like, $\phi C 31$-like, and Tuna-like viruses (purple) and their similarities represented by the Mauve output guide tree. The M. catarrhalis prophages are grouped into four clades $($ red $=$ clade 1 , blue $=$ clade 2 , orange $=$ clade 3 , green $=$ clade 4 )

Virfam Type 1 Siphoviridae from Virfam Clusters 1, 3, 4 or 5 (Additional file 2: Table S1) [18]. All prophages from clades 2, 3, and 4 were categorised as Type 1 , Cluster 3 Siphoviridae, with similarity to phages D3, HK97 and HK022, with the exception of Mcat28, which was categorised as a Type 1, Cluster 1 Siphoviridae most similar to the SPP1 group of phages. The prophages of clade 1 were more variably categorised, with Mcat1, Mcat2, Mcat3, Mcat4, Mcat7, and Mcat9 being categorised as Type 1, Cluster 3 Siphoviridae most similar to prophage $\phi 4795$; Mcat 5 was categorised as a Type 1, Cluster 4 Siphoviridae most similar to phage PBl1; Mcat6 was categorised as a Type 1, Cluster 1 Siphoviridae most similar to the SPP1 group of phages; and Mcat8 was categorised as a Type1, Cluster 5 Siphoviridae similar to phages such as $\phi C 31, \phi B T 1$, and $\phi$ HSIC.

Alignment of the $M$. catarrhalis prophages of each clade showed that clades 2,3 , and 4 were well conserved with regards to the sequence identity of phage-related genes, overall phage genomes, and synteny of genes (Additional file 3: Figure S2). Clade 1 was shown to be more variable, especially the prophages Mcat7, Mcat8, and Mcat9 (Fig. 2).
The synteny of putative $M$. catarrhalis prophages is very similar to that of the five reference genera: from proximal to distal, the phage genes are encoded in the same direction, comprising coat protein genes with associated packaging proteins, particularly terminase and portal protein genes, followed by the structural genes encoding tail sheath, tail fibre, and plate or endolysin. Additionally, the integrase gene is located at either end of the prophage. The $M$. catarrhalis clade 1 prophages are characterised by a proximal integrase, followed by the terminase, coat, then portal genes. Clade 2 prophages are different in that the integrase is located distally and is in the reverse coding direction compared to other genes. Clade 3 and 4 prophages have a distal, inverted integrase, subsequently encoding the terminase genes, portal gene, followed by coat genes.

\section{Analysis of prophage genes}

The following categories of genes were identified using PHAST, and used for analysis: coat proteins, integrases, plate proteins, portal proteins, tail (sheath) proteins, tail fibre proteins, and terminase proteins (Additional file 4: Table S2). Additionally, proteases were also identified, 


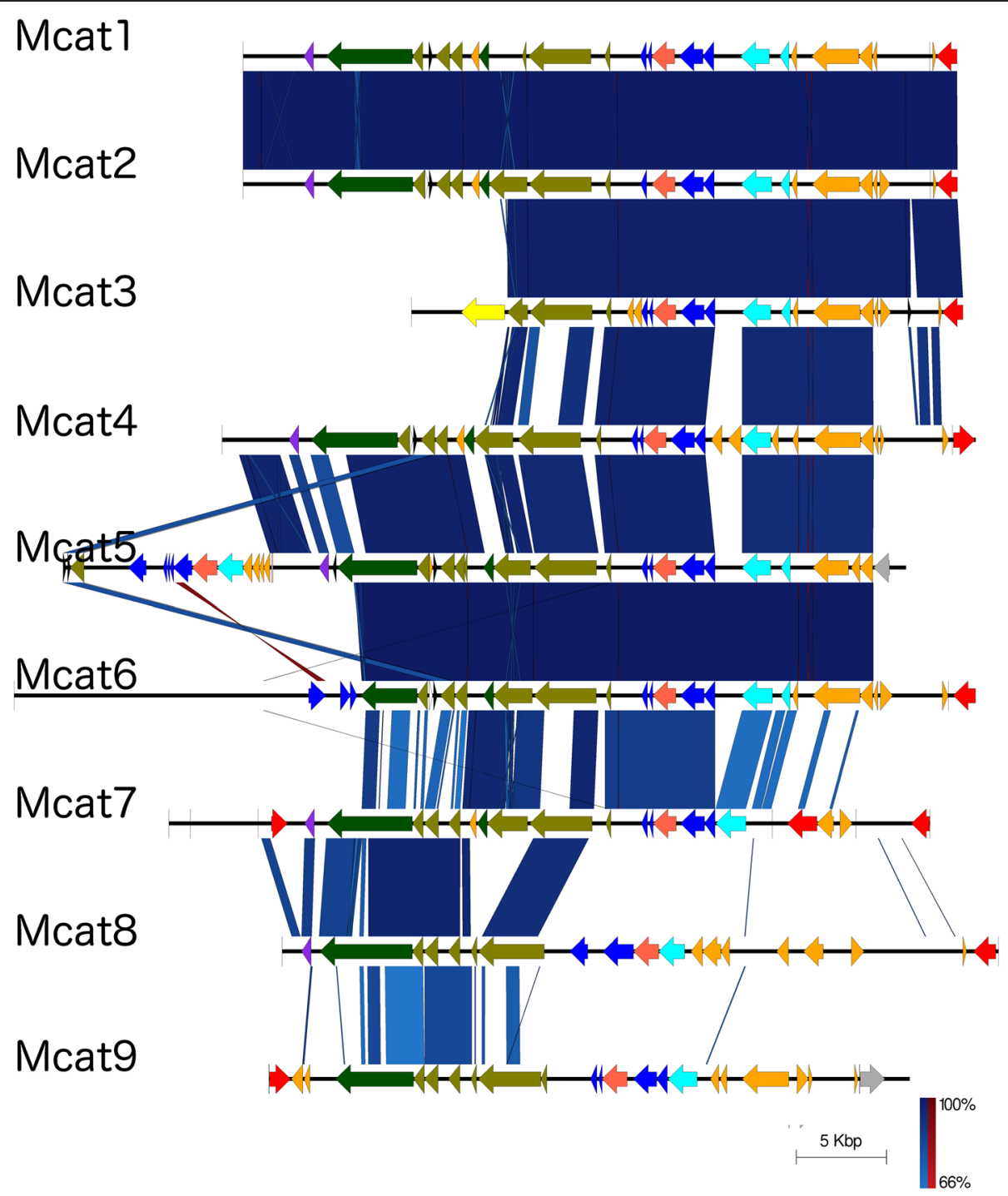

Fig. 2 Alignment of $M$. catarrhalis prophages in clade 1. Phage-related genes are shown as arrows to indicate their direction relative to the prophage sequence, and are colour coded as follows: integrase (red), terminase (light blue), coat (dark blue), portal (dark orange), tail shaft (light green), tail fibre (dark green), plate (purple), and protease (yellow). Vertical blue lines between prophages show conserved areas without inversion, and red lines between prophages show conserved areas with inversion; the intensities of these lines correspond to percent identity between adjacent prophages as indicated by the scale (lower right)

but were not used for prophage comparison because only two were identified.

Highly conserved phage-related genes: integrase, plate, portal, terminase and tail fibre genes

The integrase, plate, portal and terminase genes of $M$. catarrhalis prophages are highly conserved, and a diversity of less than 0.1 substitutions per nucleotide position was used to differentiate between gene clades. In the case of tail fibre genes, a cut-off value of about 0.4 substitutions per nucleotide position yielded better resolution and discrimination power for the clade classification (Fig. 3).
These similarity trees show that the phage-related genes of integrase, portal, large terminase subunit and small terminase subunit were distributed according to a trend that indicates a single class of such genes for the clade 1 prophages, which is separate from the respective genes from prophages in clades 2,3 , and 4 . This trend was not observed for the plate genes, which seemed to be uniformly distributed for all $M$. catarrhalis prophage classes, and the tail fibre genes, which did not seem to follow any pattern based on prophage clade (Fig. 3). All diversities represented in these trees were confirmed by inferring selective pressure using non-synonymous to synonymous ratios $\left(d_{N} / d_{S}\right)$, which confirm hyperconservation 


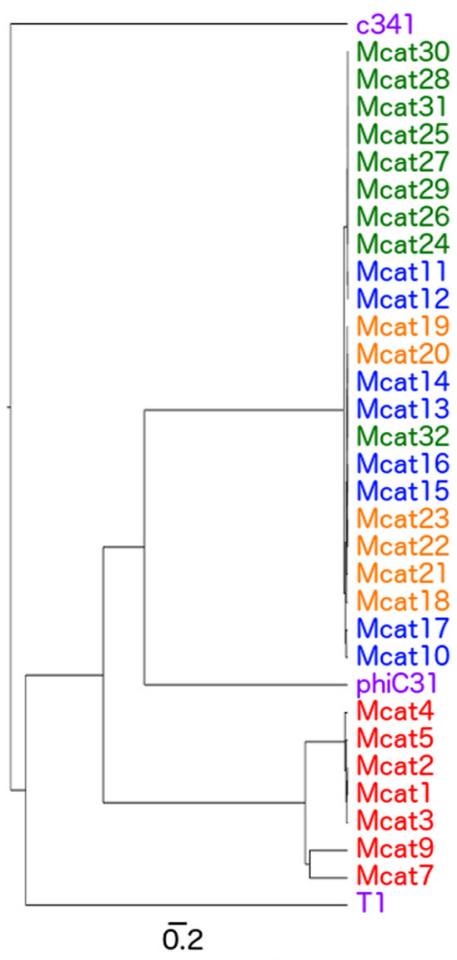

A)Terminase large subunit

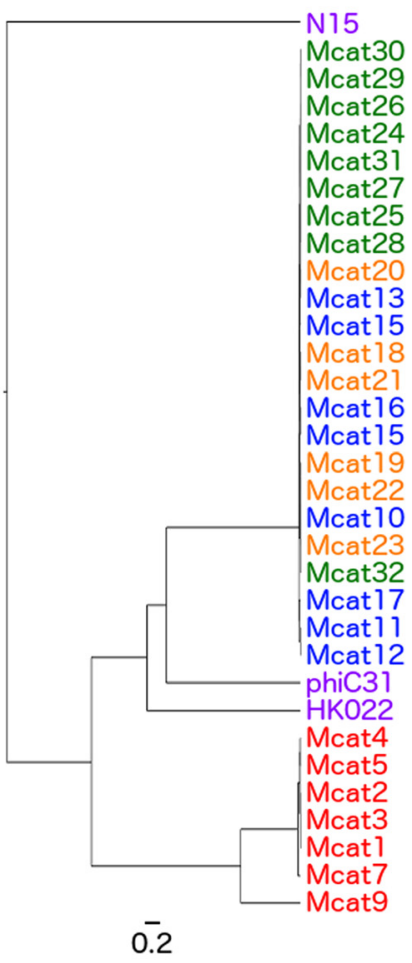

D)Portal

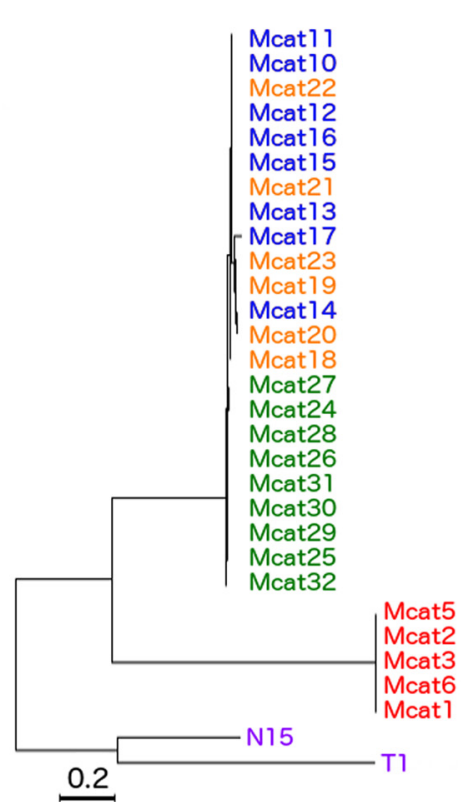

B)Terminase small subunit
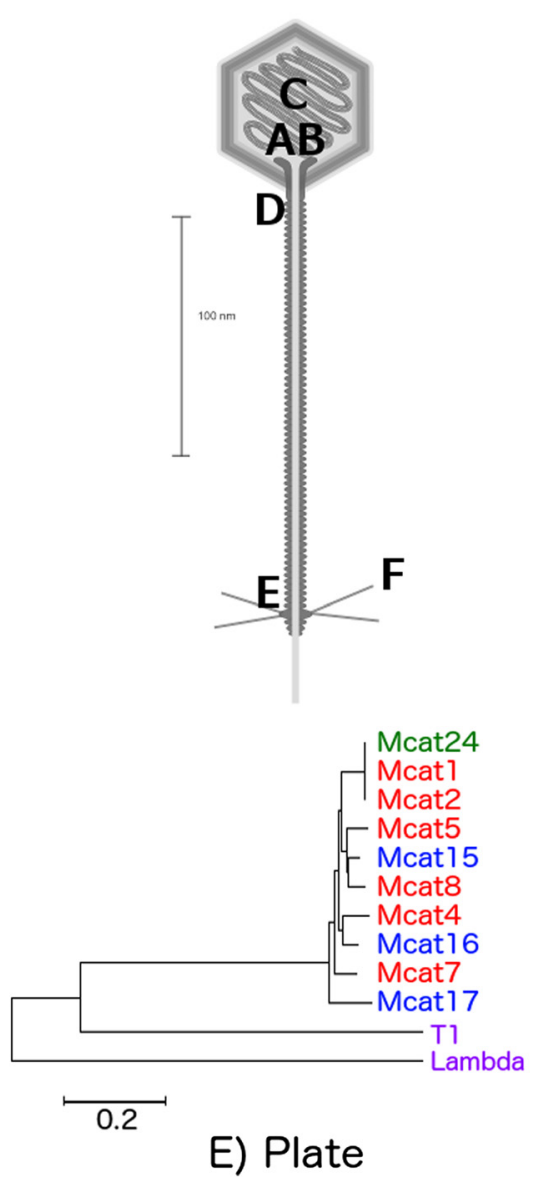

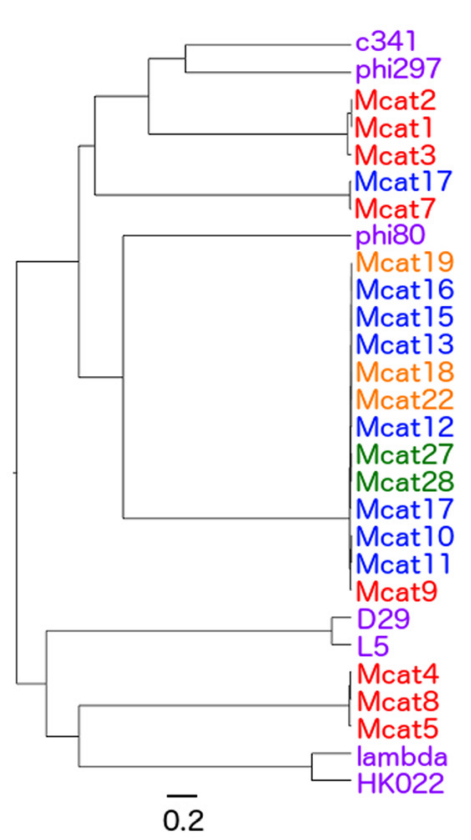

C)Integrase

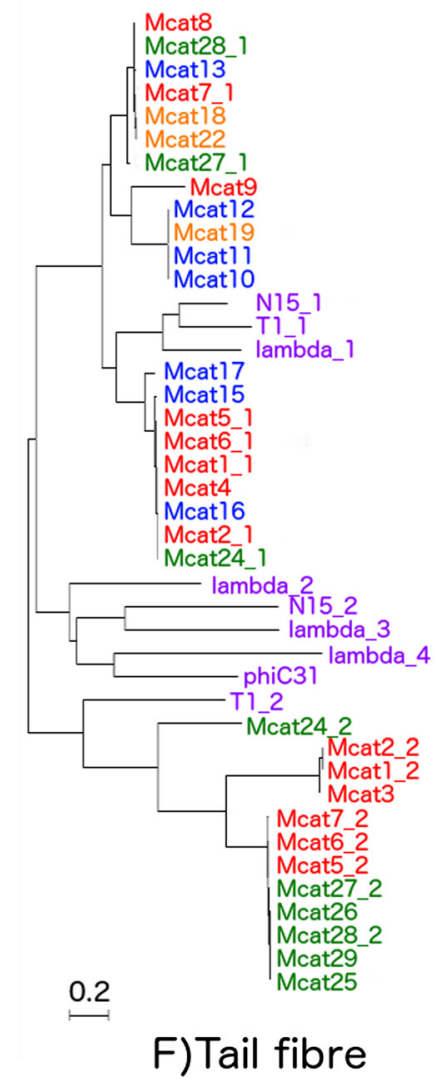

Fig. 3 (See legend on next page.) 
(See figure on previous page.)

Fig. 3 Phage-related gene analysis of $M$. catarrhalis prophages. Distance trees displaying the diversity of phage-related genes in $M$. catarrhalis prophages. a Large terminase subunit. b Small terminase subunit. $\mathbf{c}$ Integrase. $\mathbf{d}$ Portal. e Plate. $\mathbf{f}$ Tail Fibre. The $M$. catarrhalis prophage names are coloured according to clade assignment in Fig. 1; red = clade 1, blue = clade 2, orange=clade 3, green = clade 4. Reference phage genes, as detailed in the methods section, are coloured purple. Scale at lower left of each panel corresponds to 0.2 nucleotide substitutions per site. The image of a Siphoviridae phage presented in the centre with $100 \mathrm{~nm}$ scale [66] is labelled according to the analysed genes. The a terminase large subunit, $\mathbf{b}$ terminase small subunit, and $\mathbf{c}$ integrase proteins are found inside the capsid, whereas the $\mathbf{d}$ portal, e plate, and $\mathbf{f}$ tail fibre are structural

of the integrase, terminase, and portal genes (Fig. 4). Each gene displayed an average $\mathrm{d}_{\mathrm{N}} / \mathrm{d}_{\mathrm{S}}$ of $0.431,0.313$, and 0.301 respectively, which corresponds to Yates's corrected $\chi^{2}$ values of $45.2,83.8$, and 76.8 , all of which are statistically significant to the $5 \%$ level for a one-tailed test using the null hypothesis that $d_{N} / d_{S}$ values are representative of neutral evolution $\left(\mathrm{H}_{0}: \mathrm{d}_{\mathrm{N}} / \mathrm{d}_{\mathrm{S}}=1\right)$. These values are also statistically significant to the $5 \%$ level when compared to the average $\mathrm{d}_{\mathrm{N}} / \mathrm{d}_{\mathrm{S}}$ value of $M$. catarrhalis MLST housekeeping genes, 0.84 (Fig. 4).

\section{Phage-related genes of high diversity: coat and tail shaft proteins}

The coat and tail shaft genes followed a very complex distribution, because there is more than one gene of each category for each analysed phage. An analysis of the translated nucleotides was used instead, allowing for manual alignment of codons.

In the case of coat genes, comparing the generated clusters with genes of known function in the reference Siphoviridae phages revealed seven clusters of genes, four of whose functions could be inferred. These four clusters were grouped according to the following functions: coat adaptor proteins, joining and completion proteases, coat connector proteins, and head proteases. Of the remaining three clusters, only one cluster had a significant size, but with no known reference genes. Another cluster contained two $\lambda$ coat genes involved in packaging and head-tail joining. The last cluster consisted of a Mcat7 protein and a Mcat9 coat protease (Additional file 5: Figure S3).

Tail shaft genes were more diverse than coat genes, and using a translated nucleotide alignment, some genes from Mcat29, Mcat28 and Mcat25 were so divergent that they returned negative bootstrap values when aligned by ClustalW. Including these data points, up to 13 clusters were found (though some were single data points), of which only five were significant. Four clusters contained reference genes from $\lambda, \mathrm{T} 1, \mathrm{~N} 15, \phi C 31$, and D29; of which one cluster contained genes from only these reference phages, without data points from any $M$. catarrhalis prophages. One of the clusters contained only $M$. catarrhalis prophage genes without any reference genes included. Unlike the coat genes, no significant pattern was observed in the tail shaft gene distribution.

\section{Putative virulence-related genes}

A variety of potentially virulence-related genes were identified in the $M$. catarrhalis prophages (Table 2). The gene encoding virulence-associated protein $\mathrm{E}$ (VapE) was identified in Mcat19 and Mcat23. An antitoxin component highly related to hicB, the antitoxin component in the toxin-antitoxin pair hic $A B$ [19], was identified in nine prophages. Further analysis showed that one major clade of antitoxin genes is found amongst the M. catarrhalis prophage antitoxins. Additionally, the prophage Mcat5 harbours up to three putative antitoxin genes (Fig. 5). The antitoxin components identified in our prophages were queried against all the $M$. catarrhalis genomes to look for hicB homologues (Fig. 5). The hicB homologue similar to BAP37890.1 was found 81 times in the 95 genomes, of which six were in complete prophage regions. These genes were found almost exclusively in the clade 1 prophages. The exception is the hicB homologue of the antitoxin in phage Mcat24 (clade 4 prophage), which was identified 83 times. Additionally, a truncated homologue of hicB, which is found in the prophages Mcat10, Mcat11, and Mcat12 (clade 2 prophages), was identified 24 times. An ORF corresponding to tellurium resistance and another ORF corresponding to a $\mathrm{RecB}$ exonuclease were identified.

Two different protease genes were identified in Mcat3 and Mcat21. These proteases contained non-specific domains of varied functions, which are also found in a variety of organisms. The Mcat3 protease contains an ATPase associated with diverse cellular activities (AAA) domain, and two Lon domains. The Mcat21 protease contains a domain of unknown function, a structural PDZ domain, and an S41 serine endopeptidase domain; all of which are located in a Prc multi-domain region.

\section{Pan-genome analysis of $M$. catarrhalis prophages}

The pan-genome analysis of $M$. catarrhalis prophages is shown in Fig. 6. The innermost circle is the generated reference pan-genome against which each prophage genome is compared. Each concentric ring represents an individual prophage colour-coded according to the clade of origin as described above (clade $1=$ red, clade $2=$ blue, clade 3 = yellow / orange, and clade 4 = green, with each colour gradually fading as the BLAST identity score reaches $90 \%)$. The reference pan-genome was found to be 283,622 bp in length, and describes a core-genome 


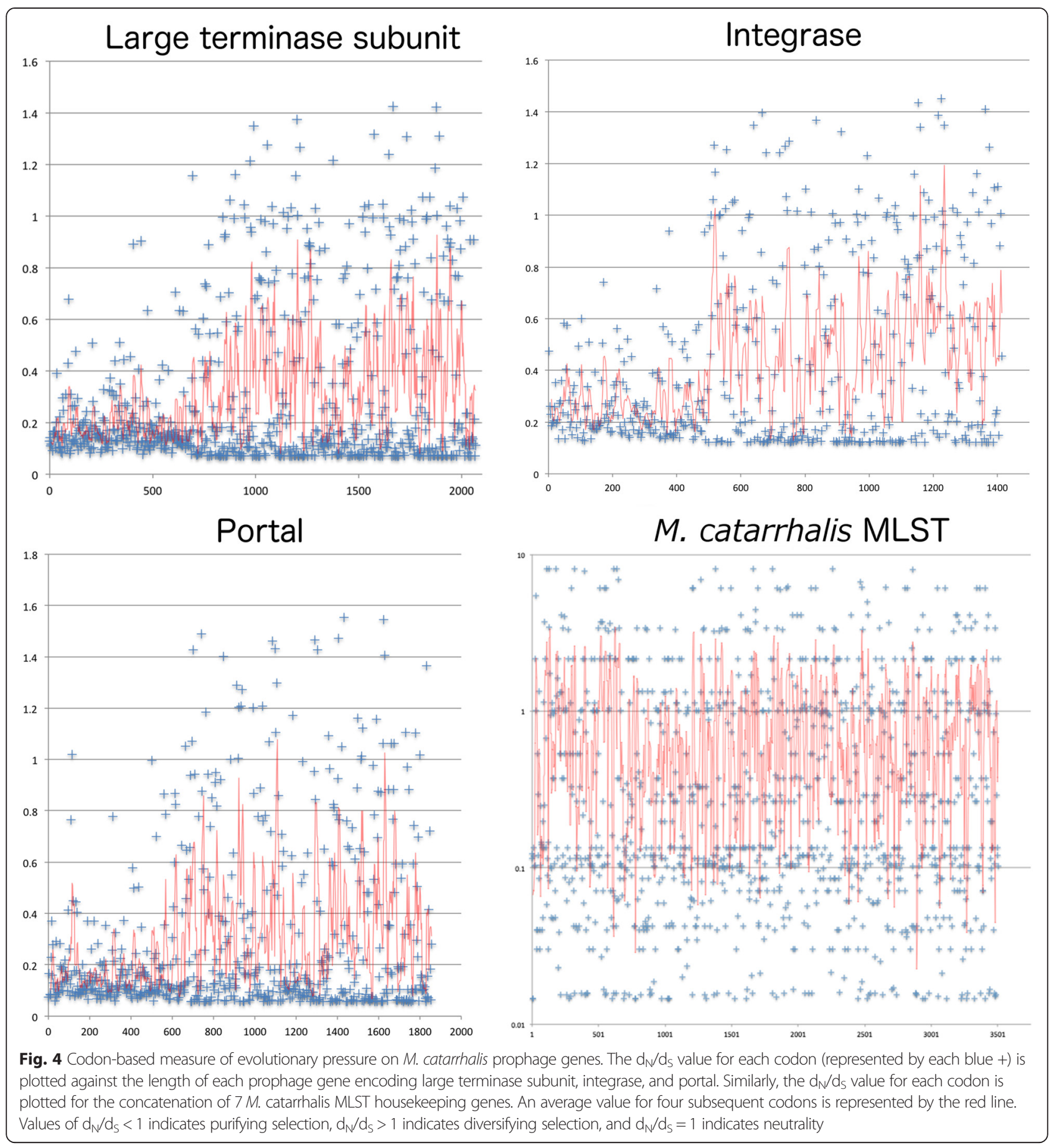

found in the majority of prophages, as well as an accessory genome more unique to individual prophages. The regions found in multiple rings (prophages) were compared to the PHAST annotation and were manually curated using BLAST against the NCBI database. They were found to code for various phage-related proteins, some virulence-related genes (as described above) or domains of unknown functions (data not shown).

\section{Distribution of prophages on a M. catarrhalis MLST backbone}

M. catarrhalis strains have previously been grouped into two clades: a serosensitive lineage that is susceptible to serum complement and a seroresistant lineage that is not [9]. This study added strain data to the MLST backbone that shows the divergence of these two inferred phenotypic lineages, and plotted the presence of 
Table 2 Putative virulence elements in M. catarrhalis prophages

\begin{tabular}{|c|c|c|c|c|c|}
\hline Prophage & Putative virulence element & Query coverage & E-value & Identity & Accession number \\
\hline Mcat1 & Toxin-antitoxin system antitoxin component HicB [Acinetobacter guillouiae] & $94 \%$ & $1 e-21$ & $62 \%$ & BAP37890.1 \\
\hline Mcat2 & Toxin-antitoxin system antitoxin component HicB [Acinetobacter guillouiae] & $94 \%$ & $1 e-21$ & $62 \%$ & BAP37890.1 \\
\hline Mcat3 & ATP-dependent protease La [Psychrobacter sp. 1501(2011)] & $97 \%$ & 0.0 & $72 \%$ & WP_007395663.1 \\
\hline Mcat4 & Toxin-antitoxin system antitoxin component HicB [Acinetobacter guillouiae] & $85 \%$ & $4 e-21$ & $66 \%$ & BAP37890.1 \\
\hline \multirow[t]{3}{*}{ Mcat5 } & Toxin-antitoxin system antitoxin component HicB [Acinetobacter guillouiae] & $94 \%$ & $1 e-21$ & $62 \%$ & BAP37890.1 \\
\hline & Antitoxin HicB [Yersinia pseudotuberculosis] & $100 \%$ & $2 e-09$ & $49 \%$ & AIN15103.1 \\
\hline & Toxin-antitoxin system antitoxin component HicB [Acinetobacter guillouiae] & $100 \%$ & $2 \mathrm{e}-20$ & $65 \%$ & BAP37890.1 \\
\hline Mcat6 & Toxin-antitoxin system antitoxin component HicB [Acinetobacter guillouiae] & $94 \%$ & $1 e-21$ & $62 \%$ & BAP37890.1 \\
\hline Mcat10 & Toxin-antitoxin system antitoxin component HicB [Acinetobacter guillouiae] & $98 \%$ & $1 e-18$ & $56 \%$ & BAP37890.1 \\
\hline Mcat11 & Toxin-antitoxin system antitoxin component HicB [Acinetobacter guillouiae] & $98 \%$ & $1 e-18$ & $56 \%$ & BAP37890.1 \\
\hline Mcat12 & Toxin-antitoxin system antitoxin component HicB [Acinetobacter guillouiae] & $98 \%$ & $1 e-18$ & $56 \%$ & BAP37890.1 \\
\hline Mcat17 & Virulence-associated E family protein [Nostoc punctiforme] & $85 \%$ & $4 e-05$ & $41 \%$ & YP_001869297.1 \\
\hline Mcat21 & Peptidase S41 [Psychrobacter sp. G] & $99 \%$ & 0.0 & $62 \%$ & YP_008162250.1 \\
\hline \multirow[t]{2}{*}{ Mcat23 } & Virulence-associated E family protein [Nostoc punctiforme PCC 73102] & $85 \%$ & $4 e-05$ & $41 \%$ & WP_012412295.1 \\
\hline & Putative RecB exonuclease [Acinetobacter phage IME_AB3] & $62 \%$ & $2 \mathrm{e}-06$ & $52 \%$ & YP_009008520.1 \\
\hline Mcat24 & Toxin-antitoxin system antitoxin component HicB [Acinetobacter guillouiae] & $94 \%$ & $1 e-21$ & $62 \%$ & BAP37890.1 \\
\hline Mcat26 & $\begin{array}{l}\text { Integral membrane protein TerC family protein [Helicobacter pylori] involved in } \\
\text { Te resistance }\end{array}$ & $100 \%$ & $2 e-36$ & $96 \%$ & WP_001934959.1 \\
\hline Mcat27 & Type III restriction endonuclease subunit R [Moraxella catarrhalis] & $100 \%$ & $3 e-41$ & $100 \%$ & WP_004463103.1 \\
\hline
\end{tabular}

Putative virulence elements identified from $32 \mathrm{M}$. catarrhalis prophages. The listed virulence element and associated accession number is taken from the BLASTP result with highest BLAST score

prophages onto the MLST backbone. The results indicated that the $M$. catarrhalis strains that host prophages are distributed randomly throughout this MLST backbone (Fig. 7).

\section{Discussion}

Bacteriophages are the most abundant biological entities in the biosphere, infecting virtually every bacterial genus and species [20]. In environmental samples, phages are found in an order of magnitude more frequently than their hosts [21]. In light of their abundance and diversity, it is interesting that no $M$. catarrhalis prophages thus far have been identified. Preliminary work on the bacterium suggested the possibility of inducible prophages in $M$. catarrhalis strains (unpublished), however, attempts to induce and isolate $M$. catarrhalis phages were unsuccessful, leading to the choice of a bioinformatics approach to identifying $M$. catarrhalis prophages. Other studies have utilised bioinformatics approaches in the annotation of prophages in a diverse range of hosts, such as Streptococcus suis [16], Mycobacterium sp. [22], and Lawsonia intracellularis [23].
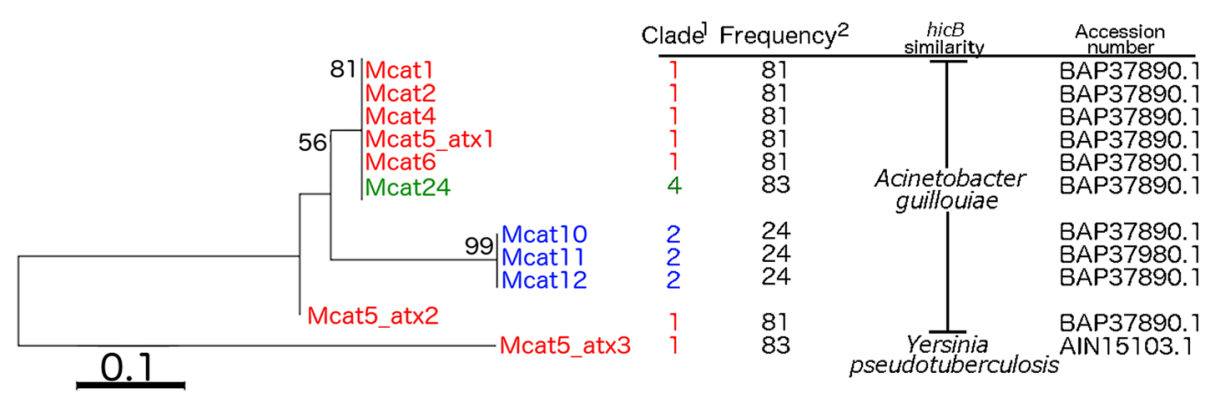

Fig. 5 Similarity of antitoxins found in M. catarrhalis prophages. Eleven antitoxin components were identified in 9 M. catarrhalis prophages with their putative evolutionary relationship depicted above. 'The antitoxins are found in prophages contained in clades 1 (red), 2 (green), and 4 (blue). ${ }^{2}$ The frequency at which the respective antitoxins or analogues can be found throughout the studied $95 \mathrm{M}$. catarrhalis genomes. The numbers above each branch in the distance tree represent the bootstrap percentage, and the scale in the lower left corner corresponds to 0.1 nucleotide substitutions per site 


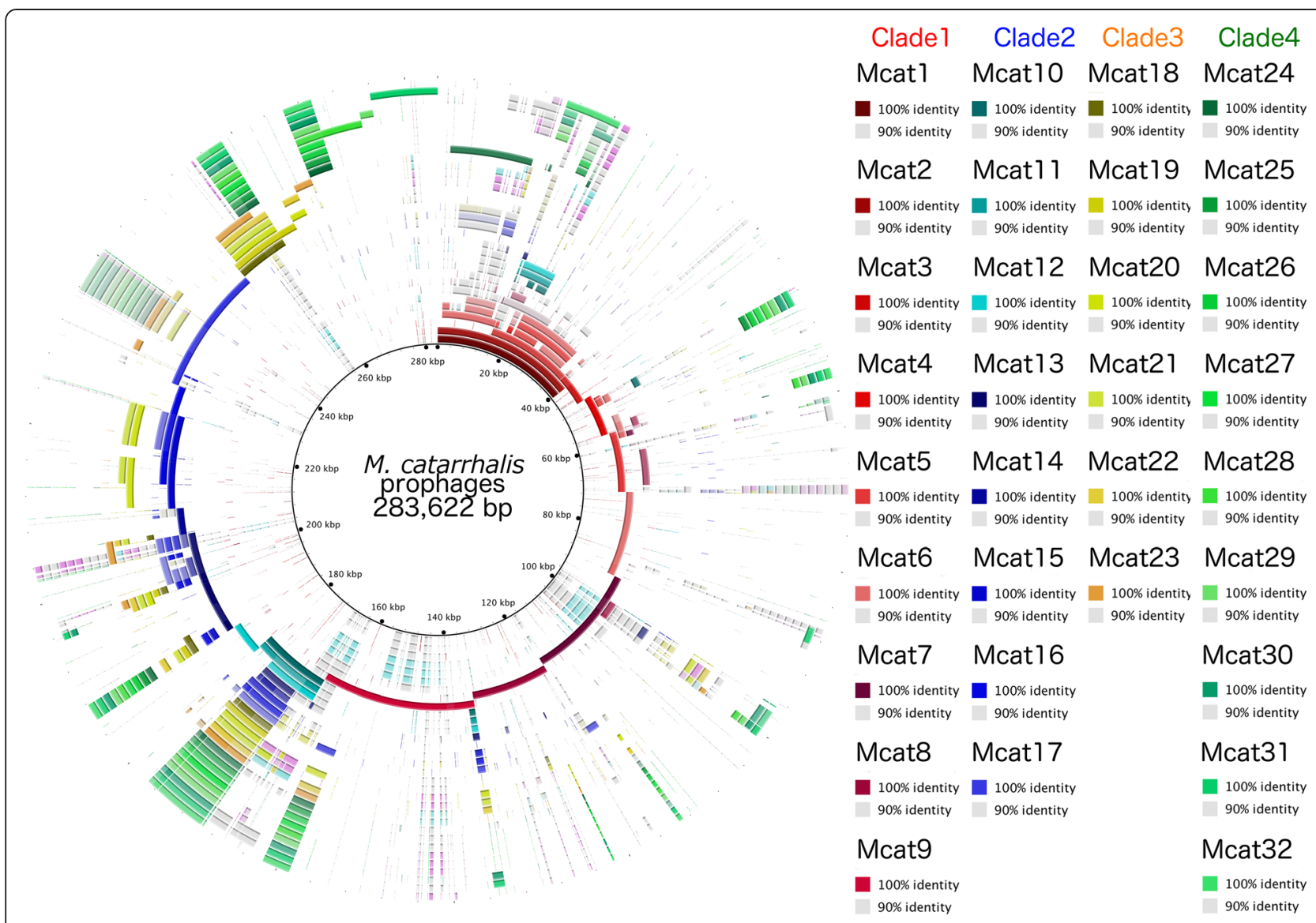

Fig. 6 Pan-genome analysis of M. catarrhalis prophages. The pan-genome of 32 M. catarrhalis prophages is 283,622 bp in length, and is represented in the BRIG image above as the central black ring. Each concentric circle outwards from the reference pan-genome represents an individual prophage colour coded according to the assigned clades (red = clade 1, blue = clade 2, orange and yellow $=$ clade 3 , green = clade 4). A percentage identity cut-off above $90 \%$ was used, which is represented by different hues in each ring (minimum of $90 \%=$ grey, and up to maximum of $100 \%=$ colour assigned to specific ring and prophage)

Analysis of $95 M$. catarrhalis assembled draft whole genome sequences resulted in the identification of 32 putative complete prophages, as well as numerous incomplete prophage remnants. These prophages, which have been assigned to the Siphoviridae family based on gene synteny and sequence identity, are annotated as 'complete' prophages according to the scoring system adopted by PHAST [24]. However, it is still unknown if they can excise from host genomes, form functional virions, and infect new host cells.

What qualifies as a prophage element has no definitive answer, as there is no comprehensive database for such elements, and no one gene is found in all prophages to serve as an identifying marker. However, it is expected that most bacterial genomes contain prophage elements, whether they are active prophages or remnants $[15,20$, $25,26]$. Additionally, as prophage elements are continuously under degradative pressure [27], it was expected that there would be present more incomplete prophage elements, than questionable prophages, than complete prophages; even though the prophage remnants may still play roles in host function [28]. M. catarrhalis, with evolution spanning about $70 \mathrm{Ma}$, would be expected to have acquired, lost, modified, and inactivated a host of genetic parasites, including phages [9]. This order of expectation was found to be true over all the samples (131 incomplete prophages, 54 questionable prophages, and 32 complete prophages). Additionally, no lysogen harboured more than one prophage, as the presence of a complete prophage confers immunity to superinfection by other similar prophages $[29,30]$. All the queried $M$. catarrhalis genomes had at least one incomplete prophage element except for the strains O35E, and 2041717D.

The discovered $M$. catarrhalis prophages fall into a narrow range of sizes ranging from $25 \mathrm{~kb}$ to $55 \mathrm{~kb}$; Siphoviridae viruses have genome sizes usually ranging from 35 to $70 \mathrm{~kb}$ of DNA, with a mean of about $50 \mathrm{~kb}$. The average GC content of the identified prophages was found to be $41.90 \%$, which is similar to that of the $M$. catarrhalis genome ( 41\%). This is different from the 


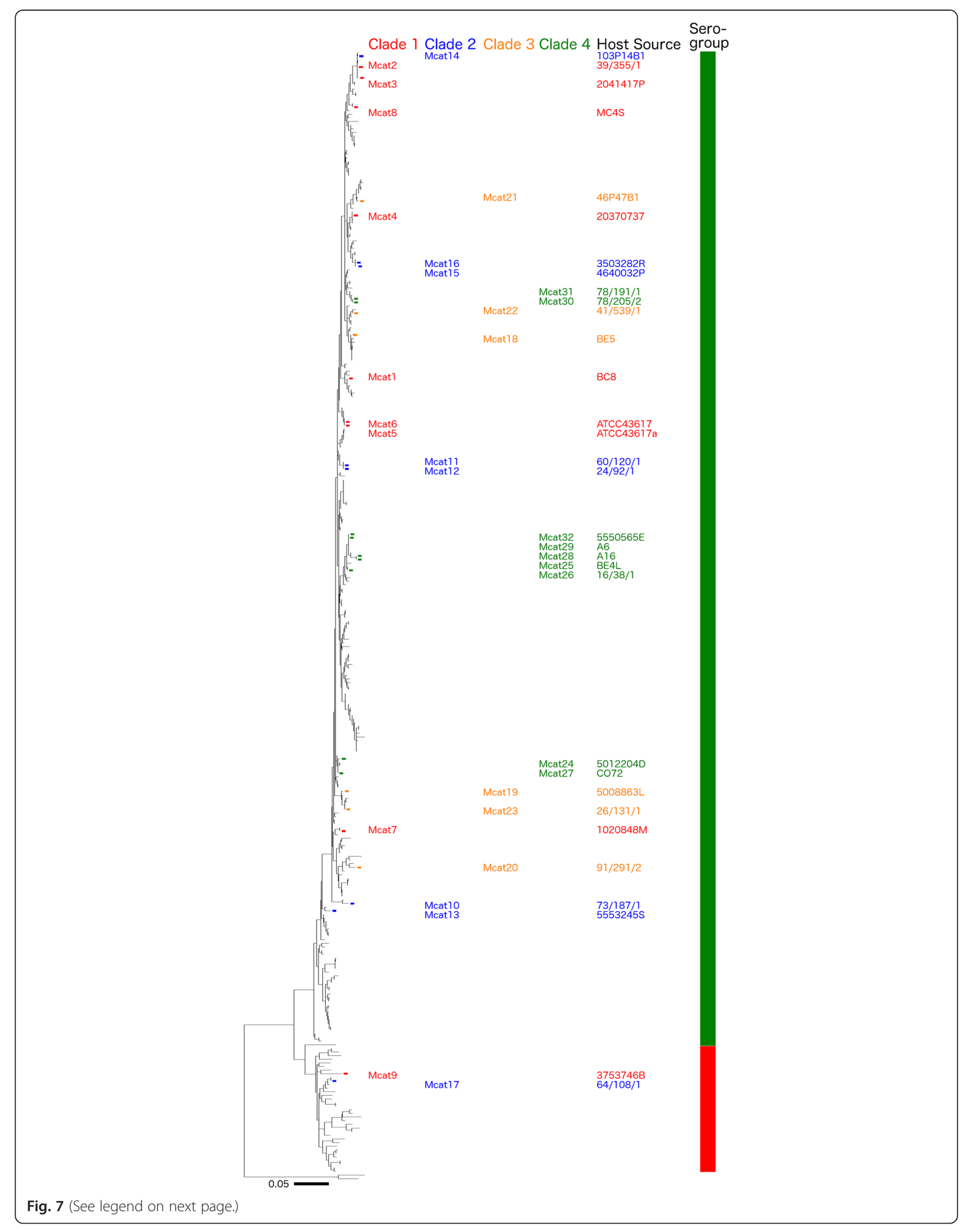


(See figure on previous page.)

Fig. 7 Distribution of prophages and prophage hosts on M. catarrhalis MLST phylogenetic tree. The phylogenetic tree based on M. catarrhalis MLST data is shown on the left. The two putative sero-groups based on MLST data are shown on the right where green=seroresistant and red = serosensitive. Each $M$. catarhalis strain that hosts a complete prophage as described in this study is labelled according to colour of the prophage clade, red = prophage from clade 1 , blue $=$ clade 2 , orange $=$ clade 3 , and green = clade 4 . The scale on the lower left of image is equal to 0.05 nucleotide substitutions per site

average GC content of Siphoviridae (roughly $52.28 \%$, calculated from all 685 Siphoviridae sequences available in the NCBI database at the time of publication). However, the similarity of GC contents between identified prophages and host $M$. catarrhalis may be indicative of the prophages having incorporated into $M$. catarrhalis genomes early in the host's speciation, or that the prophages have lain dormant in the host genomes.

An alignment of the putative attachment sites clusters them into a major and six minor groups (Additional file 6: Figure S4.). The clustering of the att sites did not correspond to the prophage clades, and did not directly reflect the diversity of integrase genes. However, it is known that various integrases can have secondary attachment sites that are divergent from the sequence of the primary attachment site, which may explain the variety of putative att sites [31-33]. Though the att sites are not identical, the conservation of a putative core site may relate to the hyperconservation of integrase genes [34-36].

Categorisation of prophages using global sequence and protein similarity has previously yielded reproducible and consistent results [37-39]. However, the reliability of such analyses has only been previously reported in the Podoviridae and Myoviridae families. This study found that a similar alignment and comparison approach classified $32 \mathrm{M}$. catarrhalis prophages as Siphoviridae, within a distinct group. Our prophage classification was reinforced by the analysis of head-neck-tail proteins using the programme Virfam [18]. The diversity of $M$. catarrhalis prophages is demonstrated in the breadth of categorisation using this software, where all prophages were categorised into four different Clusters of the Type I Siphoviridae (Additional file 2: Table S1).

The multiple alignment of all $32 \mathrm{M}$. catarrhalis putative prophages divides them into four separate clades. Based on the synteny and similarity of genes, prophage clades 2,3 , and 4 are highly related, but remain distinct, whereas clade 1 is more divergent than the others. This is illustrated by Virfam prophage categorisation, where 22 of 23 prophages in clades 2, 3, and 4 were categorised as Type 1, Cluster 3 Siphoviridae with high similarity to phages D3, HK97 and HK022 (the exception being Mcat28, which was categorised as a Type 1, Cluster 1 Siphoviridae). The clade 1 prophages were more variable, as described in the results section. Thus, clades 2,
3 , and 4 represent a homogeneous and conserved group of $M$. catarhalis prophages, but clade 1 represents a less well-defined cluster of $M$. catarrhalis prophages. Addition of more prophages to the distance tree would illustrate whether clade 1 requires further subdivision into more clades, or if a single clade is sufficient, particularly if the core genome for clade 1 prophages is smaller, or less homogeneous than that of clades 2, 3, and 4 .

The BRIG alignment shown in Fig. 6 indicates that the prophages from clades 2,3 , and 4 are highly similar in their core genomes, and the core genome of clade 2 is a subset of that of clade 3, which in turn is a subset of the core genome of clade 4 . The clade 1 prophages have a nearly unique core genome, represented by the span $0-$ $40 \mathrm{~kb}$ of the reference pan-genome, which comprises the prophages Mcat1 - Mcat7. Mcat7, Mcat8 and Mcat9 are prophages with similarities to the clade 1 prophages, but also to the prophages of clades 2, 3, and 4, though they do not share the core genomes of the latter 3 clades.

The inclusion of five reference genera into the distance tree shows that the clades are adequately different to be considered for the categorisation of $M$. catarrhalis prophages; and that these prophages share some homology with the five genera of phages, but remain distinct as a group of their own. It is interesting to note that the highest region of identity between the reference genera and the $M$. catarrhalis prophages is centred around the tail fibre genes, which are involved in host recognition. We speculate that this may be a mechanism that confers host specificity to the phages.

This result is supported by the analysis of the more divergent genes for coat and tail shaft proteins. Coat genes clustered based on function into clusters representing 1) adaptor proteins, 2) joining and completion proteases, 3) connector proteins, 4) head proteases, and two undefined clusters, where there was little diversity in each cluster (Additional file 5: Figure S3). This distribution was observed for all $M$. catarrhalis prophages, regardless of prophage clade, indicating that selective pressure for the coat genes is different from that upon the integrase, terminase, portal, and plate genes. As with most structural genes, genetic mutation is more permissible than in non-structural genes, and as long as the function of the protein is preserved, there can be a larger amount of diversity before the gene renders its virus non-viable. Analysis of the tail shaft genes, however, presents a 
different picture. Although there are 7 clusters of genes, there are many more outlier genes or groups of genes that are significantly divergent from the major groups. Additionally, none of these groups could be assigned according to function, as there was no description of tail shaft genes in the reference genera. Furthermore, the distribution of genes from the reference genera puts these genes in closely associated clusters, with two of the 7 clusters of tail shaft genes consisting almost exclusively of genes from the reference genera. These distributions demonstrate diversity in $M$. catarrhalis prophage structural genes, which is expected for such genes under low selective pressure over a long evolutionary time period, such as that suggested by the analysis below.

The distribution of prophages and their M. catarrhalis hosts were compared based on the MLST tree created from 312 strains. There was no observable pattern correlating the prophage distribution and MLST backbone, which leads to the conclusion that the identified prophages are not confined to or fixed within a particular lineage of $M$. catarrhalis host. Furthermore, analysis of the prophage clades and how they are distributed amongst their hosts indicates that there is no observable pattern or relationship between prophage clade and $M$. catarrhalis host strain (Fig. 7). The data in this study suggest that the prophages have evolved alongside $M$. catarrhalis host before the divergence of serosensitive and seroresistant lineages, and that lateral transfer of the prophages between hosts is not limited by the divergence of the two M. catarrhalis lineages. The seroresistant lineage harbours prophages from all four prophage clades, but the serosensitive lineage only harbours prophages from clades 1 and 3. However, it is important to note that the serosensitive group is much smaller than the seroresistant group, and is only populated by 2 prophage hosts (as opposed to the 30 in the seroresistant group). This is compounded by the sequencing bias of this study in favour of the seroresistant lineage, where only four of the 95 isolates were found to be of the serosensitive lineage. The absence of any discernible pattern in this analysis demonstrates the diversity of $M$. catarrhalis prophages, supporting the prophage genetic analysis. However, further studies are required to establish any relationship between $M$. catarrhalis prophage and host, especially pertaining to $M$. catarrhalis isolates from the serosensitive lineage.

This diversity of $M$. catarrhalis prophages is contrasted by a hyperconservation of the phage specific genes encoding integrase, portal, and terminase (large and small subunits), which follows a general trend where the genes for clade 1 prophages cluster together, whereas those of clades 2, 3, and 4 cluster together (Fig. 3). This supports the argument that the prophages of clades 2, 3, and 4 share a relatively recent common ancestor, which is also reflected in the homogeneity of their core genome; whereas clade 1 prophages seem to have branched off earlier than clades 2, 3, and 4. Although the core genome of the clade 1 prophages shows a higher degree of diversity (Fig. 2 and Fig. 6), it is interesting that the integrase, portal, and terminase genes of the group 1 prophages are hyperconserved. This may support that the clade 1 prophages be categorised together, regardless of the near-absence of a core genome. The portal and two terminase genes can be grouped together as genes involved in packaging of new viral genetic material into a newly formed capsid. The generalised conservation of non-structural and portal genes in the two groups (clade 1 prophages compared to clade 2,3 , and 4 prophages) may indicate a difference in physiology of the two groups. This hypothetical evolutionary divergence is not reflected in the plate genes, which exhibit almost no diversity. This may be because the plate protein is essential for the injection of phage genetic material into the host, and significant mutation in the plate gene may render mutants incapable of infection and propagation. However, only 10 plate sequences were isolated, and it could be that this small sample size is indicative of plate genes that are too divergent from the database queries to be identified.

The integrase protein is spanned by two Pfam domains: DUF4102 and Phage_integrase, with the latter containing the active site. Further analysis of the ratio of non-synonymous to synonymous codons, $\mathrm{d}_{\mathrm{N}} / \mathrm{d}_{\mathrm{S}}$ values (also known as $\omega$ or $K_{a} / K_{s}$ ), and the trend of $d_{N} / d_{S}$ values averaged over 4 codons, for the integrase gene (Fig. 4) showed that the N-termini of the integrase genes (corresponding to DUF4102) are hyperconserved, with $\mathrm{d}_{\mathrm{N}} / \mathrm{d}_{\mathrm{S}}$ trends between 0.2 and 0.4 , whereas the distal two thirds of the genes show conservation, where the $d_{N} / d_{S}$ trend only exceeds 1.0 at three points. Similar analysis of the large terminase subunit and portal genes revealed that the $d_{N} / d_{S}$ trend lines, averaged over 4 codons, for all genes are statistically significantly below 1.0, supporting a hypothesis of purifying selection for these genes. Such conservation suggests that the non-structural genes play a central and stable role in the maintenance of the phage.

The putative cargo genes identified in M. catarrhalis prophages are indirectly related to virulence. The majority of ORFs are related to antitoxin components of toxin-antitoxin systems, of which the most descriptive BLAST hit is hicB, the antitoxin component of the hic $A B$ toxin-antitoxin system [19], and these antitoxins are most highly related to the hicB components in Acinetobacter guillouaie (BAP37890.1) and Yersinia pseudotuberculosis (AIN15103.1). Toxin-antitoxin systems have been shown to be involved in bacterial defence against 
phage infection via different mechanisms [40-42]. HicA expression is bacteriostatic, mediated by HicA cleavage of mRNA [43]. The HicAB toxin-antitoxin system is a type II system, where the HicB protein interacts sterically with HicA to inhibit its action. The corresponding toxin genes with sequence identity to hicA (BAP37889.1 and AIN15869.1) were not found using the BLAST and MUMmer [44] alignment algorithms. A Smith-Waterman alignment of the toxin and antitoxin components revealed that they share significant sequence similarity, but the relatively short toxin lengths may obscure results, leading to a reduced number of significant hits. Based on the presence of hicB antitoxin homologues in up to $87.4 \%$ of the sequenced strains, it is suggested that at least one toxinantitoxin system is utilised in $M$. catarrhalis, possibly relating to defence against phages. However, it could be that these are remnants of such systems, and have become degraded. Further research into the potential toxin-antitoxin systems related to $M$. catarrhalis prophages is under way.

Two prophages, Mcat23 and Mcat17 harboured genes similar to vapE. The virulence associated protein (Vap) family of virulence genes has been associated with clinically virulent strains of bacteria. The function of these virulence genes is not well described, but it is known that VapA is required in conjunction with other Vap proteins [45] in Rhodococcus equi for diversion of the phagosome-maturation pathway and prevents acidification of phagosomes [46]. With the exception of VapF, the Vap proteins are well conserved on the amino acid and nucleic acid levels, and share a well-conserved Cterminus [45, 47]. Although vapE is described to be present with $v a p F$ in virulence plasmids [48], vap genes have been shown to be widely distributed and also occur chromosomally as single genes [47].

\section{Conclusions}

In conclusion, the approach used in this study has resulted in the first identification and characterisation of prophages in $M$. catarrhalis. The presence of prophages in host genomes does not prove functionality as active phages, but the distribution of similar prophages across different host lineages suggests that the prophages were, and may still be active. Induction of the prophages will be required to prove that active virions can be produced. This study demonstrates that along with significant diversity in $M$. catarrhalis prophages, there is unexpected hyperconservation of phage non-structural, as well as portal genes, which suggests that the preservation of these genes confers a physiological advantage and affects the fitness of $M$. catarrhalis host. The hyperconservation of phage-related genes may also indicate common ancestral (pro)phages for the M. catarrhalis prophages of clades 2, 3, and 4, as well as a common ancestral (pro)phage for the clade 1 prophages, which co-evolved with the bacterial species. Additionally, it is demonstrated that the prophages harbour virulence-associated genes, potentially playing a role in the physiology and virulence of M. catarrhalis.

\section{Methods \\ Collection, sequencing and assembly of $M$. catarrhalis genomes}

Eighty-five $M$. catarrhalis strains were collected for sequencing and analysis in this project, and an additional 12 sequences were obtained from the NCBI database (http://www.ncbi.nlm.nih.gov/) [15], detailed in Table 1. Forty-one strains were obtained from PathWest Laboratory Medicine WA, Australia; thirty-three strains were from the Kalgoorlie Otitis Media Research Project (KOMRP), Australia $[49,50] ; 3$ strains were from the Alexander Project [51]; 3 commensal strains were from Taiwan; three strains were from elsewhere in Australia; 1 strain was from Belgium; and 1 strain was from Holland [52]. This study included bacterial isolates for which no corresponding patient data were used. There is no requirement under Australian law to seek consent for the use of anonymised bacterial isolates for research. The strains were grown overnight on Brain Heart Infusion Agar (Oxoid) or Blood Agar (PathWest Media, P018) at $37^{\circ} \mathrm{C}$, and stored for up to 2 weeks at $4{ }^{\circ} \mathrm{C}$. Whole plates of bacterial lawns were suspended in $180 \mu \mathrm{l}$ ATL Buffer, and subsequently treated according to the Qiagen DNeasy blood \& tissue protocol for purification of total DNA from animal tissues (spin-column protocol; Qiagen, Venlo). All strains were sequenced according to the Illumina Nextera-XT protocol, Revision C (Illumina, San Diego) [53]. This protocol was modified, where instead of using the Normalization Beads for the library normalisation step, the libraries were manually normalised using a 1:3 diluted library on an Agilent Technologies 2100 Bioanalyzer (Agilent Technologies, California) using a High Sensitivity DNA chip according to the Illumina Nextera (Illumina, San Diego) protocol [54]. This was performed to achieve a more uniform library index. Deep sequencing of the genomes was performed on an Illumina MiSeq sequencer (Illumina, San Diego) using 250 bp pair-end read chemistry and assembled de novo using the software Spades [55], where k-mer selection was optimised automatically.

\section{Identification of $M$. catarrhalis prophages}

Prophages were identified in $M$. catarrhalis assembled genome sequences using the automated annotation programme PHAST [24]. PHAST assigns a score to putative prophages based on the presence of 'cornerstone' genes, phage-related genes, as well as the length of these genes. Putative prophages scoring between 90 and 150 are denoted as complete prophages, whereas those 
scoring between 60 and 90 are denoted questionable prophages, and those scoring below 60 are denoted incomplete prophages. Each ORF coding for phage-related genes was manually curated using BLASTP [56]. Complete prophage sequences were extracted from their respective $M$. catarrhalis genomes. ORFs 100 bp or larger were identified using Artemis, and selected for those opening with typical and atypical start codons [57]. These ORFs were uploaded as protein sequences to Virfam for prophage classification by analysis of phage-related proteins, and in particular head-necktail module genes [18].

\section{M. catarrhalis prophage similarity}

All complete prophage genomes were diverse, such that they could not be aligned by simple nucleotide comparison. Instead, a less stringent block alignment was created using progressiveMauve [58]. A guide tree was visualised from the Mauve distance matrix using the programme FigTree 1.4.0. Regions of sequence identity between $M$. catarrhalis prophages, as well as archetypes from other Caudovirales phages were identified using the BLAST algorithm [59] from EasyFig 2.1 [60]. Additionally, each of the prophages that formed a clade were aligned and visualised using the BLAST algorithm from EasyFig 2.1 to show regions of sequence identity.

\section{Analysis of phage-related genes}

Phage-related genes with similar functional annotation identified by PHAST were extracted from their respective prophage genomes, and aligned using ClustalW via MEGA 6.06 [61] and Unipro UGENE [62]. The M. catarrhalis prophage genes were compared to homologous genes in the five reference genera, $\lambda, \mathrm{L} 5, \mathrm{~N} 15, \phi \mathrm{C} 31$ and Tuna viruses, as well as homologous genes from closely related species: c341, $\$ 297, \phi 80$, and HK022. The nucleotide alignments of conserved genes were used for construction of distance trees using the Maximum Likelihood method using MEGA 6.06 with the following parameters: bootstrap value of 1000, Tamura-Nei model, a uniform rate of substitution amongst sites, and inferred using Nearest-Neighbor-Interchange heuristic method applied to an initial Neighbor-Joining/BioNJ tree. These trees were confirmed using the Bayesian analysis of the MrBayes [63] utility available in Unipro UGENE, in which a substitution model of HKY85 was used (Nst $=2)$ with default settings and a random seed number. For genes too diverse to be aligned and compared at the nucleotide level, translated amino acid alignments were also used, and this was constructed using the ClustalW feature of Unipro UGENE. For genes too divergent to be aligned via Maximum Likelihood method (coat and tail genes), translated protein alignments were used to construct Neighbor-Joining trees in Unipro UGENE using the following parameters; a distance matrix model of F84, transition/transversion ratio of 2.0, and a bootstrap (replicate) value of 1000. These trees were confirmed using the Bayesian analysis of the MrBayes utility available in Unipro UGENE, in which a substitution model of HKY85 was used (Nst $=2)$ with default settings and a random seed number [63]. The per codon $d_{N} / d_{S}$ ratios $(\omega)$ for the integrase, large terminase and portal genes were computed as a by-product of creating Bayesian phylogenetic trees from the respective genes using MrBayes [63], where $d_{N} / d_{S}$ was allowed to vary across codons. A gamma model was used for variation between sites, together with a proportion of invariant sites. Mean $\mathrm{d}_{\mathrm{N}} / \mathrm{d}_{\mathrm{S}}$ values were reported for each triplet and then input to Excel to create the graph, including trend-line.

\section{Pan-genome analysis of $M$. catarrhalis prophages}

The sequences for all $M$. catarrhalis prophages were used for a pan-genome comparison. To create a reference pan-genome of the prophages, the programme Panseq was run utilising default parameters [64]. Each prophage genome was aligned against this reference genome using the BLAST [56] algorithm found with BLAST Ring Image Generator (BRIG) [65] with the following parameters: all $M$. catarrhalis prophages represented a unique ring, except for the annotations for Mcat5 and Mcat6, from strains ATCC43617 and ATCC43617a, which were combined into a single output to avoid redundancy; an identity threshold between 90 and $100 \%$ was selected for the image colour gradient generation; a default minimum threshold of $50 \%$ was used for alignment; phages were ordered and colour coded according to their respective clades as described in the results section; and the output image was scaled to include a legend.

\section{Extraction of multi-locus sequence type backbone}

MLST data for eight housekeeping genes, $a b c Z, a d k, e f p$, fum $C, \operatorname{gly} \beta, \operatorname{mut} Y$, $p p a$, and $\operatorname{trpE}$, were extracted from the assembled genomes. The gene sequences were concatenated and the concatenation was used to construct a phylogenetic tree similar to that performed by Wirth et al. in 2007 [9]. The sequences were aligned and a tree was created using MEGA6.06 using the following parameters: a Nearest-Neighbour Interchange model was imposed on an initial Neighbour Joining $(\mathrm{NJ} / \mathrm{BioNJ})$ tree to create a Maximum-Likelihood tree modelled on protein-coding nucleotide sequences, with a bootstrap value of 1000, using a Tamura-Nei model, and a uniform rate of substitution amongst sites [61]. The dataset of MLST genes and strains includes 312 strains of $M$. catarrhalis, of which 95 strains are from this study, 12 from Davie et al. [15], and the remainder from Wirth et al. [9]. 


\section{Additional files}

Additional file 1: Figure S1. PHAST annotation of $32 \mathrm{M}$. catarrhalis prophages. $32 \mathrm{M}$. catarrhalis prophages were annotated as complete prophages by the PHAST programme, defined as such for scoring between 90 and 150 on the PHAST scoring system. Each arrow represents an open reading frame found in each prophage, colour coded as in the legend below each column: tail fibre (dark green), terminase (light blue), coat (dark blue), integrase (red), plate (purple), portal (dark orange), tail shaft (light green), hypothetical protein (navy blue), and other phage-like protein (light orange). Each prophage is named according to the methods section, and this name is colour coded according to the prophage clade of origin as per Fig. 1: clade 1 (red), clade 2 (dark blue), clade 3 (orange), and clade 4 (dark green). (TIFF 6959 kb)

Additional file 2: Table S1. PHAST and Virfam results for $32 \mathrm{M}$. catarrhalis prophages. ${ }^{1} 32 \mathrm{M}$. catarrhalis prophages were annotated as complete prophages by the PHAST programme, defined as such for scoring between 90 and 150 on the PHAST scoring system. ${ }^{2}$ Virfam analysis for categorisation of the prophages designated a family, type, and cluster for each prophage, along with a phylogenetic tree depicting the relationship of the $M$. catarrhalis prophage head-neck-tail modules with those of known phages in the Aclame database. (DOCX $143 \mathrm{~kb}$ )

Additional file 3: Figure S2. Alignment of $M$. catarrhalis prophages of clade 2 (Mcat10-Mcat17, dark blue), clade 3 (Mcat18-Mcat23, orange), and clade 4 (Mcat24-Mcat32, dark green). Each horizontal line represents a prophage, and each arrow represents an open reading frame (ORF) colour coded according to function: terminase (light blue), portal (dark orange), protease (yellow), coat (dark blue), tail shaft (light green), integrase (red), other phage-related protein (light orange), tail fibre (dark green), and plate (purple). Vertical lines between prophages shows aligned regions whose hues of grey correspond to the percentage identity of aligned regions as specified in the spectrum at the bottom right of each image. Image generated by EasyFig [60]. (TIFF 4381 kb)

Additional file 4: Table S2. Presence of phage-related genes in analysed $M$. catarrhalis prophages. The detection of phage-related genes in analysed $M$. catarrhalis prophages using the programme PHAST is signified with presence $(+)$ or absence $(-)$. ${ }^{1}$ The assignment of prophages to clades 1 to 4 is as described in methods section. (DOCX $122 \mathrm{~kb}$ )

Additional file 5: Figure S3. Distance tree of translated coat nucleotide sequences of $M$. catarrhalis prophages. Six groups are identified, four of which are clustered according to function: (red) head adaptor protein, (dark blue) joining and completion protease, (dark green) connector protein, and (purple) head protease. Alignment was constructed using ClustalW algorithm, and tree generation with Neighbor-Joining method using Unipro UGENE programme. The schematic of a Siphoviridae phage is presented on the right with labels for phage-related structures and 100nm scale [66]. (TIFF $141 \mathrm{~kb}$ )

Additional file 6: Figure S4. Clustering of $M$. catarrhalis prophage attachment sites. Twenty-three att sites are identified, which cluster into a major group and 6 minor groups. Each att site is labelled according to prophage of origin, which is coloured according to the relevant prophage clade in Fig. 1 (red $=$ clade 1 , dark blue $=$ clade 2 , orange $=$ clade 3 , and green $=$ clade 4$)$. The nucleotide alignment is shown on the right (green $\mathrm{A}=$ adenine, red $\mathrm{T}=$ thymine, blue $\mathrm{C}=$ cytosine, purple $\mathrm{G}=$ guanine), and scale at bottom left indicates 10 nucleotide substitutions per site. (TIFF $3747 \mathrm{~kb}$ )

\section{Competing interests}

The authors declare that they have no competing interests.

\section{Authors' contributions}

AA performed the sequencing of genomes, and bioinformatics analysis of studied genomes. CMK and BJC provided advice and supervision throughout the project. MJW and TTP were involved with bioinformatics analysis, as well as providing advice and supervision throughout the project. CYT and FT were involved in the sequencing of genomes. All authors have read and approved the final manuscript.

\section{Acknowledgements}

We would like to acknowledge Clin/A/Prof. Deborah Lehmann (Telethon Kids Institute) and Ms Jacinta Bowman (PathWest Laboratory Medicine WA) who have kindly helped in the collection of $M$. catarrhalis strains. TTP is supported by the NHMRC Grant ID572723 awarded to Barry J Marshall.

\section{Author details}

${ }^{1}$ School of Pathology and Laboratory Medicine, The University of Western Australia, Perth, WA, Australia. ${ }^{2}$ School of Chemistry and Biochemistry, The University of Western Australia, Perth, WA, Australia.

\section{Received: 3 July 2015 Accepted: 16 October 2015}

\section{Published online: 24 October 2015}

\section{References}

1. Murphy TF, Parameswaran GI. Moraxella catarrhalis, a human respiratory tract pathogen. Clin Infect Dis. 2009;49:124-31.

2. Verduin $\mathrm{CM}, \mathrm{Hol}$ C, Fleer A, Van-Dijk H, Van-Belkum A. Moraxella catarrhalis: from emerging to established pathogen. Clin Microbiol Rev. 2002;15(1):125-44

3. Melendez PR, Johnson RH. Bacteremia and septic arthritis caused by Moraxella catarrhalis. Rev Infect Dis. 1991;13:428-9.

4. Ionannidis JPA, Worthington M, Griffiths JK, Snydman DR. Spectrum and significance of bacteremia due to Moraxella catarrhalis. Clin Infect Dis. 1995;21:390-7.

5. Jin Y. Moraxella catarrhalis meningitis: a case report. Chin Med J. 2000;113(4):381-2.

6. Verduin CM, Jansze M, Verhoef J, Fleer A, Dijk H. Complement resistance in Moraxella (Branhamella) catarrhalis is mediated by a vitronectin-binding surface protein. Clin Exp Immunol. 1994;97:50.

7. Verduin $\mathrm{CM}, \mathrm{Hol}$ C, van Dijke E, Faber JAJ, Jansze M, Verhoef J, et al. Assessment of complement-mediated killing of Moraxella (Branhamella) catarrhalis isolates by a simple method. Clin Diagn Lab Immunol. 1995;2(3):365-8.

8. Verduin CM, Jansze M, Hol C, Mollnes TE, Verhoef J, Dijk H. Differences in complement activation between complement-resistant and complementsensitive Moraxella (Branhamella) catarrhalis strains occur at the level of membrane attack complex formation. Infect Immun. 1994;62(2):589-95.

9. Wirth T, Morelli G, Kusecek B, van Belkum A, van der Schee C, Meyer A, et al. The rise and spread of a new pathogen: seroresistant Moraxella catarrhalis. Genet Res. 2007;17:1647-56.

10. Chen F, Lu J. Genomic sequence and evolution of marine cyanophage P60: a new insight on lytic and lysogenic phages. Appl Environ Microbiol. 2002;68(5):2589-94.

11. Abedon ST, LeJeune JT. Why bacteriophage encode exotoxins and other virulence factors. Evol Bioinformatics Online. 2005;1:97-110.

12. O'Brien AD, Newland JW, Miller SF, Holmes RK, Smith HW, Formal SB. Shigalike toxin-converting phages from Escherichia coli strains that cause hemorrhagic colitis or infantile diarrhea. Science. 1984;226(4675):694-6.

13. Bobay L-M, Touchon M, Rocha EPC. Pervasive domestication of defective prophages by bacteria. Proc Natl Acad Sci U S A. 2014;111(33):12127-32.

14. de Paepe M, Hutinet G, Son O, Amarir-Bouhram J, Schbath S, Petit M-A. Temperate phages acquire DNA from defective prophages by relaxed homologous recombination: the role of Rad52-like recombinases. Public Libr Sci: Genet. 2014;10(3), e1004181.

15. Davie JJ, Earl J, de Vries SPW, Ahmed A, Hu FZ, Bootsma HJ, et al. Comparative analysis and supragenome modeling of twelve Moraxella catarrhalis clinical isolates. BMC Genomics. 2011;12(70):1-7.

16. Tang F, Bossers A, Harders F, Lu C, Smith H. Comparative genomic analysis of twelve Streptococcus suis (Pro)phages. Genomics. 2013;101:336-44.

17. Bootsma HJ, van Dijk H, Verhoef J, Fleer A, Mooi FR. Molecular characterization of the BRO $\beta$-lactamase of Moraxella (Branhamella) catarrhalis. Antimicrob Agents Chemother. 1996;40:966-72.

18. Lopes A, Tavares P, Petit M-A, Guerois R, Zinn-Justin S. Automated classification of tailed bacteriophages according to their neck organization. BMC Genomics. 2014;15:1027-43.

19. Makarova KS, Grishin NV, Koonin EV. The HicAB cassette, a putative novel, RNA-targeting toxin-antitoxin system in archaea and bacteria. Bioinformatics. 2006;22(21):2581-4.

20. Clokie MRJ, Millard AD, Letarov AV, Heaphy S. Phages in nature. Bacteriophage. 2011;1(1):31-45. 
21. Fuhrman JA. Marine virueses and their biogeochemical and ecological effects. Nature. 1999;399(10):541-8.

22. Fan X, Xie L, Li W, Xie J. Prophage-like elements present in Mycobacterium genomes. BMC Genomics. 2014;15:243. doi:10.1186/1471-2164-15-243.

23. Vannucci FA, Kelley MR, Gebhart CJ. Comparative genome sequencing identifies a prophage-associated genomic island linked to host adaptation of Lawsonia intracellularis infections. Vet Res. 2013;44:49.

24. Zhou Y, Liang Y, Lynch KH, Dennis JJ, Wishart DS. PHAST: a fast phage search tool. Nucleic Acids Res. 2011;41(6):1-6.

25. Canchaya C, Proux C, Fournous G, Bruttin A, Brussow H. Prophage genomics. Microbiol Mol Biol Rev. 2003;67(2):238-76.

26. Roodt Y, Bragg RR, Albertyn J. Identification of prophages and prophage remnants within the genome of Avibacterium paragallinarum bacterium. Sequencing. 2012. doi:10.1155/2012/953609.

27. Golais F, Holly J, Vitkovska J. Coevolution of bacteria and their viruses. Folia Microbiologica. 2013;58:177-86.

28. Wang G-H, Niu L-M, Ma G-C, Xiao J-H, Huang D-W. Large proportion of genes in one cryptic WO prophage genome are actively and sex-specifically transcribed in a fig wasp species. BMC Genomics. 2014;15:893.

29. Fogg PCM, Allison HE, Saunders JR, McCarthy AJ. Bacteriophage lambda: a paradigm revisited. J Virol. 2010;84(13):6876-9.

30. Berngruber TW, Weissing FJ, Gandon S. Inhibition of superinfection and the evolution of viral latency. J Virol. 2010;84(19):10200-8.

31. Campbell AM. Chromosomal insertion sites for phages and plasmids. J Bacteriol. 1992;174(23):7495-9.

32. Csorda-Toth E, Boros I, Venetianer P. Nucleotide sequence of a secondary attachment site for bacteriophage lambda on the Escherichia coli chromosome. Nucleic Acids Res. 1979;7(5):1335-41.

33. Nagaraja R, Weisberg RA. Specificity determinants in the attachment sites of bacteriophages HK022 and lambda. J Bacteriol. 1990;172(11):6540-50.

34. Dorgai L, Sloan S, Weisberg RA. Recognition of core binding sites by bacteriophage integrases. J Mol Biol. 1998;277:1059-70.

35. Groth AC, Calos MP. Phage integrases: biology and applications. J Mol Biol. 2004:335:667-78

36. Campbell AM, Ad C-C, Ginsberg ML. Specificity in DNA recognition by phage integrases. Gene. 2002;300:13-8.

37. Comeau AM, Tremblay D, Moineau S, Rattei T, Kushkina Al, Tovkach Fl, et al. Phage morphology recapitulates phylogeny: the comparative genomics of a new group of myoviruses. Public Libr Sci: One. 2012;7(7), e40102.

38. Lavigne R, Darius P, Summer EJ, Seto D, Mahadevan P, Nilsson AS, et al. Classification of Myoviridae bacteriophages using protein sequence similarity. BMC Microbiol. 2009;9:224.

39. Lavigne R, Seto D, Mahadevan P, Ackermann H-W, Kropinski AM. Unifying classical and molecular taxonomic classification: analysis of the Podoviridae using BLASTP-based tools. Res Microbiol. 2008;159:406-14.

40. Hazan R, Engelberg-Kulka H. Escherichia coli mazEF-mediated cell death as a defense mechanism that inhibits the spread of phage P1. Mol Gen Genomics. 2004;272:227-34.

41. Pecota DC, Wood TK. Exclusion of T4 phage by the hok/sok killer locus from plasmid R1. J Bacteriol. 1996;178(7):2044-50.

42. Fineran PC, Blower TR, Foulds IJ, Humphreys DP, Lilley KS, Salmond GPC. The phage abortive infection system, ToxIN, functions as a protein-RNA toxin-antitoxin pair. Proc Natl Acad Sci U S A. 2009;106(3):894-9.

43. Jorgensen MG, Pandey DP, Jaskolska M, Gerdes K. HicA of Escherichia coli defines a novel family of translational-independent mRNA interferases in bacteria and archaea. J Bacteriol. 2009;191(4):1191-9.

44. Kurtz S, Phillippy A, Delcher AL, Smoot M, Shumway M, Antonescu C, et al. Versatile and open software for comparing large genomes. Genome Biol. 2003;5(2):R12.

45. Anand T, Bera BC, Vaid RK, Shanmugasundaram K, Sharma G, Virmani N, et al. Molecular characterization of virulence-associated protein (Vap) family genes in pathogenic Rhodococcus equi isolates from clinical cases of Indian equines. Indian J Biotechnol. 2014;13:195-202.

46. von Bargen K, Polidori M, Becken U, Huth G, Prescott JF, Haas A. Rhodococcus equi virulence-associated protein $A$ is required for diversion of phagosome biogenesis but not for cytotoxicity. Infect Immun. 2009;77(12):5676-81.

47. Whittingham JL, Blagove EV, Finn CE, Luo H, Miranda-CasoLuengo R, Turkenburg JP, et al. Structure of the virulence-associated protein VapD from the intracellular pathogen Rhodococus equi. Acta Crystallographica Sec D. $2014 ; 70: 2139-51$.
48. Takai S, Hines SA, Sekizaki T, Nicholson VM, Alperin DA, Osaki M, et al. DNA sequence and comparison of virulence plasmids from Rhodococcus equi ATCC33701 and 103. Infect Immun. 2000;68(12):6840-7.

49. Lehmann D, Arumugaswamy A, Elsbury D, Finucane J, Stokes A, Monck R, et al. The Kalgoorlie Otitis Media Research Project: rationale, methods, population characteristics and ethical considerations. Paediatr Perinat Epidemiol. 2008;22:60-71.

50. Pingault NM, Bowman J, Lehmann D, Riley TV. Antimicrobial susceptibility of Moraxella catarrhalis isolated from children in Kalgoorlie-Boulder, Western Australia. Pathology. 2010;42(3):273-9.

51. Felmingham D, Gruneberg RN, Group TAP. A multicentre collaborative study of the antimicrobial susceptibility of community-acquired, lower respiratory tract pathogens 1992-1993: the Alexander project. J Antimicrob Chemother. 1996;38(Supplementary A):1-57.

52. Mollenkvist A, Nordstrom T, Hallden C, Christensen JJ, Forsgren A, Riesbeck K. The Moraxella catarrhalis immunoglobulin D-binding protein MID has conserved sequences and is regulated by a mechanism corresponding to phase variation. J Bacteriol. 2003;185(7):2285-95.

53. Illumina. Nextera XT DNA sample preparation guide, Illumina proprietary. 2012. Part\# 15031942 Rev. C.

54. Epicentre I. Nextera DNA sample prep Kit (Illumina-compatible). 2011. Cat. Nos. GA091120, GA0911-50, GA0911-96, and GABC0950.

55. Bankevich A, Nurk S, Antipov D, Gurevich AA, Dvorkin M, Alexander S, et al. SPAdes: a new genome assembly algorithm and its application to single-cell sequencing. J Comput Biol. 2012;18(5):455-77.

56. Zhang Z, Schwartz S, Wagner L, Miller W. A greedy algorithm for aligning DNA sequences. J Comput Biol. 2000;7(2):203-14

57. Rutherford K, Parkhill J, Crook J, Horsnell T, Rice P, Rajandream M-A et al. Artemis: sequence visualization and annotation. Bioinformatics. 2000;16(10):944-5.

58. Rissman Al, Mau B, Biehl BS, Darling AE, Glasner JD, Perna NT. Reordering contigs of draft genomes using the Mauve aligner. Bioinformatics. 2009;25(16):2071-3.

59. Altschul S, Gish W, Miller W, Myers E, Lipman D. Basic local alignment search tool. J Mol Biol. 1990;215(3):403-10.

60. Sullivan MJ, Petty NK, Beatson SA. EasyFig: a genome comparison visualizer. Bioinformatics. 2011;27(7):1009-10.

61. Tamura K, Stecher G, Peterson D, Filipski A, Kumar S. MEGA6: molecular evolutionary genetics analysis version 6.0. Mol Biol Evol. 2013;30(12):2725-9.

62. Okonechnikov K, Golosova O, Fursov M. Team U. Unipro UGENE: a unified bioinformatics toolkit. Bioinformatics. 2012;28(8):1166-7.

63. Ronquist $F$, Teslenko M, van der Mark P, Ayres DL, Darling AE, Hohna S, et al. MrBayes 3.2: efficient Bayesian phylogenetic inference and model choice across a large model space. Syst Biol. 2012;61(3):539-42.

64. Laing C, Buchanan C, Taboada EN, Zhang Y, Kropinski AM, Villegas A, et al. Pan-genome sequence analysis using Panseq: an online tool for the rapid analysis of core and accessory genomic regions. BMC Bioinformatics. 2010;11:461.

65. Alikhan N-F, Perry NK, Zekour NLB, Beatson SA. BLAST ring image generator (BRIG): simple prokaryote genome comparisons. BMC Genomics. 2011;12(402):1-10.

66. Gleiberg. Schematischer querschnitt durch einen lambda-phagen (virusfamilie Siphoviridae), Lambda-phage-01.png. Germany: Gleiberg; 2006. Image of Bacteriophage Lambda.

\section{Submit your next manuscript to BioMed Central and take full advantage of:}

- Convenient online submission

- Thorough peer review

- No space constraints or color figure charges

- Immediate publication on acceptance

- Inclusion in PubMed, CAS, Scopus and Google Scholar

- Research which is freely available for redistribution

Submit your manuscript at www.biomedcentral.com/submit

C Biomed Central 